Article

\title{
Prediction of Heat Transfer during Condensation in Non-Circular Channels
}

\author{
Mirza M. Shah \\ Engineering Research Associates, 10 Dahlia Lane, Redding, CT 06896, USA; mshah.erc@gmail.com; \\ Tel.: +1-860-869-1328
}

Received: 6 May 2019; Accepted: 17 June 2019; Published: 19 June 2019

\begin{abstract}
It is desirable to know whether correlations for condensation in round tubes can be used for non-circular channels. To investigate this matter, a number of well-known correlations for mini and macro channels as well as some for flattened channels were compared to a database for condensation in non-circular channels. Data included square, rectangular, triangular, semi-circular, drum, N, and W shaped channels as well as flattened tubes. The data included 15 fluids, hydraulic diameter 0.067 to $1.46 \mathrm{~mm}$, aspect ratio 0.14 to 7 , reduced pressure 0.045 to 0.77 , and mass flux from 48 to $1000 \mathrm{kgm}^{-2} \mathrm{~s}^{-1}$. None of the correlations worked well for flattened tubes. Data for all other shapes were best predicted by the Shah correlation with mean absolute deviation of $20.1 \%$ with 1120 data points from 22 sources. None of the other correlations was found satisfactory over the entire range.
\end{abstract}

Keywords: condensation; heat transfer; mini-channels; non-circular channels; correlations

\section{Introduction}

Mini channels are being widely used in heat exchangers including condensers because they offer several advantages including compact size, high heat transfer coefficients, and lower cost. They also reduce the amount of refrigerant in refrigeration systems and thus minimize environmental impact in case of leakage. Many channels of various shapes are in use or being tried. These include circular, square, rectangular, triangular, and oval. To ensure proper design, it is important to be able to predict heat transfer during condensation in channels of all shapes. Several correlations have been published which are stated to be applicable to circular as well as non-circular channels. These correlations use an equivalent diameter for application of non-circular channels; no other modification is made. Notable examples of such correlations are Kim and Mudawar [1], Dorao and Fernandino [2], and Shah [3]. On the other hand, several theoretical and experimental studies indicate that heat transfer in non-circular channels is higher than that in circular channels of the same equivalent diameter.

Wang and Rose [4-6] performed theoretical analyses considering gravity, viscous, and surface tension forces. Liquid film was assumed to be laminar. Channel shapes considered included triangle, rectangle, and square besides circular. It was found that surface tension causes liquid to collect in the corners, resulting in thinning of liquid film on the sides thus increasing heat transfer coefficients. Wen et al. [7] performed numerical investigation of condensation in three round tube and channels of aspect ratio 2 to 6 produced by flattening these tubes. Mass flux varied from 600 to $1000 \mathrm{kgm}^{-2} \mathrm{~s}^{-1}$. They found that liquid film is thick at the curved sides and thin at the flat sides. Average film thickness was smaller in flattened tubes compared to the round tubes and heat transfer increased with increasing aspect ratio. Zhang et al. [8] performed a similar study on flattened tubes made from a $3.78 \mathrm{~mm}$ diameter tube with similar results.

Wilson et al. [9] measured heat transfer during condensation of R-134a and R-410A in a horizontal tube of $8.9 \mathrm{~mm}$ and channels obtained by flattening it. They concluded that flattened tubes have higher heat transfer than round tubes. Kim et al. [10] condensed R-134a in a $5.1 \mathrm{~mm}$ diameter round tube as 
well as in flattened tubes made from it. Heat transfer coefficients in flattened tubes were found to be higher than given by round tube correlations and they therefore gave a new correlation. Darzi et al. [11] measured heat transfer coefficients during condensation of R-600a in an $8.7 \mathrm{~mm}$ diameter tube and channels obtained by flattening it. They found heat transfer coefficient to increase with increasing aspect ratio. Kaewon et al. [12] condensed R-134a in a round tube $3.51 \mathrm{~mm}$ diameter and flattened tubes made from it. They concluded that heat transfer in flattened tubes increases with increasing aspect ratio and is higher than that from round tube correlations. Solanki and Kumar [13] condensed $\mathrm{R}-134 \mathrm{a}$ in a round tube of diameter $8.91 \mathrm{~mm}$ and two channels made by flattening this tube. They also concluded that heat transfer in flattened tubes is higher than that predicted by round tube correlations. They gave a new correlation for flattened tubes. Del Col et al. [14] condensed R-134a in a square channel of $1.23 \mathrm{~mm}$ hydraulic diameter. They compared their data with the data of Matkovic et al. [15] for a $0.96 \mathrm{~mm}$ diameter round tube. They concluded that heat transfer at the lowest mass flux was higher in the square channel. They attributed this to surface tension thinning the liquid film by drawing liquid into corners. At higher flow rates, they found no difference between the round and square channels. In contrast, the CFD studies mentioned above found increase in heat transfer at high flow rates.

The theoretical and experimental studies mentioned in the foregoing suggest that correlations for round tubes are not applicable to non-circular channels. On the other hand, the correlations of Shah [3], Dorao and Fernandino [2] and Kim and Mudawar [1] were shown to agree with data for both circular and non-circular channels from numerous sources. Due to this apparent contradiction, it was felt that there was a need to further investigate this matter and the research reported here was undertaken. Data for non-circular channels were collected from many sources and compared to several general correlations as well as correlations developed specifically for non-circular channels. The results of this comparison are reported and discussed in this paper and recommendations are made for design.

In this paper, channels with hydraulic diameter $\leq 3 \mathrm{~mm}$ are considered mini channels according to the classification of Kandlikar [16]. Other classifications have been reviewed by Shah [17].

\section{Prediction Methods}

\subsection{Theoretical}

Many theoretical studies have been done in which the governing equations were solved numerically to determine heat transfer coefficient. Several of these were discussed in Section 1. Da Riva et al. [18] did a numerical simulation of condensation in a $1 \mathrm{~mm}$ diameter tube and compared its predictions with experimental data. Their finding was that the assumption of laminar liquid film gives good agreement with measurements only at low flow rates; turbulence has to be taken into consideration at higher flow rates. Mechanistic analysis of Rohsenow et al. [19] showed that in the presence of vapor shear, liquid film can become turbulent at very low Reynolds numbers. In the analysis by Kim and Mudawar [20] for condensation in a rectangular channel, liquid film became turbulent at Reynolds number of 25. This indicates that the theory of Wang and [4-6] which assumed laminar liquid film has very limited applicability.

Kharangate and Mudawar [21] reviewed the work on numerical modelling by various methods. They concluded that while much progress has been achieved, accurate and efficient methodology remains to be developed. None of the published studies have been shown to agree with varied data from many sources. Hence such methods are as yet not suitable for use in practical design.

\subsection{Correlations}

Numerous correlations have been published for heat transfer during condensation in channels. Most of them have been verified with very limited data, usually the authors' own. Such correlations fail when compared to a wider data range. However, a few general correlations have been published which were verified over a very wide range of data. Notable among those for conventional channels are Dobson and Chato [22], Thome et al. [23], Cavallini et al. [24], and Shah [25,26]. The first three 
mentioned are applicable only to horizontal tubes and use the Nusselt formula for condensation outside horizontal tubes for calculating heat transfer at low flow rates where heat transfer becomes heat flux dependent. Heat transfer on the upper part of tube is calculated with the Nusselt equation while heat transfer of liquid flowing at the bottom is calculated with a forced convection equation. These correlations require that heat flux be known for comparing them with test data. The Shah correlation uses the Nusselt formula for condensation in vertical tubes in the heat flux dependent regime and does it in a way that heat flux is not needed for comparison with test data. The Shah correlation is applicable to horizontal as well as vertical down flow while the other three are applicable only to horizontal flow.

Many correlations have been proposed specifically for minichannels. These have been reviewed by Awad et al. [27] and Del Col et al. [28]. Recent correlations for mini channels include those by Jige et al. [29], Rahman et al. [30], and Keinath and Garimella [31]. Among the numerous correlations specifically for minichannels, only that of Kim and Mudawar [1] has been validated with wide ranging data from many sources. It showed good agreement with a wide ranging database that included many fluids, horizontal and vertical up and down flow, and hydraulic diameters from 0.424 to $6.22 \mathrm{~mm}$. The data included round and rectangular single channels and multiport channels.

Two correlations have been published which have been validated with wide ranging databases that included both round and non-circular channels of mini and macro sizes. These are the correlations of Dorao and Fernandino [2] and Shah [3]. The latter is given in the following.

\subsubsection{The Shah Correlation}

Shah [32] gave a correlation applicable to both horizontal and vertical tubes of conventional diameters. It has been widely used but it is limited to moderate pressure and higher flow rates. Shah $[25,26]$ gave an improved version which is applicable over the entire range of pressures and flow rates from very low to very high. The data with which it was validated was mostly for macro size tubes. Further modified versions for application to both macro and mini channels were given in Shah $[33,34]$ and finally in Shah [3]. The [3] correlation uses the [25,26] correlation in some situations. It is also used in the correlation of Kim et al. [10] for flattened tubes. The 2013 version is therefore given first.

Shah $[25,26]$ correlation

The correlation uses the following two equations.

$$
\begin{aligned}
& h_{I}=h_{L O}\left(1+\frac{3.8}{Z^{0.95}}\right)\left(\frac{\mu_{L}}{14 \mu_{G}}\right)^{\left(0.0058+0.557 p_{r}\right)} \\
& h_{N u}=1.32 \operatorname{Re}_{L O}-1 / 3\left[\frac{\rho_{L}\left(\rho_{L}-\rho_{G}\right) g k_{L}^{3}}{\mu_{L}{ }^{2}}\right]^{1 / 3}
\end{aligned}
$$

Equation (2) is the Nusselt equation for condensation in vertical tubes, with the constant multiplied by 1.2 as recommended by McAdams [35].

This correlation has three regimes, I, II, and III.

In Regime I,

$$
h_{T P}=h_{I}
$$

In Regime II,

$$
h_{T P}=h_{I}+h_{N u}
$$

In Regime III:

$$
h_{T P}=h_{N u}
$$

$\mathrm{h}_{\mathrm{LO}}$ in Equation (1) is the heat transfer coefficient of the liquid phase flowing alone in the tube. It is calculated by the following equation:

$$
h_{L O}=0.023 \operatorname{Re}_{L O}^{0.8} \operatorname{Pr}_{L}^{0.4} k_{L} / D
$$


The heat transfer regimes are determined as follows.

Horizontal Tubes:

Regime I occurs when:

$$
J_{g} \geq 0.98(Z+0.263)^{-0.62}
$$

Regime III occurs when:

$$
J_{g} \leq 0.95\left(1.254+2.27 Z^{1.249}\right)^{-1}
$$

If neither of the above conditions is satisfied, it is Regime II.

$\mathrm{J}_{\mathrm{g}}$ is the dimensionless vapor velocity defined as:

$$
J_{g}=\frac{x G}{\left(g D \rho_{G}\left(\rho_{L}-\rho_{G}\right)\right)^{0.5}}
$$

Equation (8) for the boundary of Regime III was given in Shah [26]. In Shah [25], Regime II occurred when Equation (7) was not satisfied.

Vertical Downflow:

Regime I occurs when

$$
J_{g} \geq \frac{1}{2.4 Z+0.73}
$$

Regime III prevails when:

$$
J_{g} \leq 0.89-0.93 \exp \left(-0.087 Z^{-1.17}\right)
$$

If the Regime is not determined to be I or III by Equations (10) and (11), it is Regime II.

Shah [3] correlation

Heat transfer regimes are the same as in Shah $[25,26]$ correlation for both horizontal and vertical downflow if any of the following conditions are applicable:

- Fluid is a hydrocarbon, Regime is I and $\mathrm{p}_{\mathrm{r}}<0.4$

- $\quad$ Fluid is hydrocarbon and Regime is III

- $\operatorname{Re}_{\mathrm{LT}}<100$

If any of the above conditions is fulfilled, the Shah $[25,26]$ correlation is to be used.

If none of the above conditions is applicable, heat transfer regimes are determined as follows.

Horizontal Flow

Regime I occurs if $\mathrm{We}_{\mathrm{GT}}>100$ and $\mathrm{Fr}_{\mathrm{LT}}>0.012$ and:

$$
J_{g} \geq 0.98(Z+0.263)^{-0.62}
$$

Regime III occurs if $\mathrm{Fr}_{\mathrm{L}}>0.012$ and:

$$
J_{g} \leq 0.95\left(1.254+2.27 Z^{1.249}\right)^{-1}
$$

If it is not Regime I or III, it is Regime II.

Vertical Downflow

Regime I occurs when $\mathrm{We}_{\mathrm{GT}}>100$ and:

$$
J_{g} \geq \frac{1}{2.4 Z+0.73}
$$

Regime III occurs when:

$$
J_{g} \leq 0.89-0.93 \exp \left(-0.087 Z^{-1.17}\right)
$$

If it is not Regime I or III according to Equations (14) and (15), it is Regime II. 
Further Equation (1) is used when $\mathrm{D}_{\mathrm{HYD}}>3 \mathrm{~mm}$. When $\mathrm{D}_{\mathrm{HYD}} \leq 3 \mathrm{~mm}$, the following equation given by Cavallini et al. [24] is used.

$$
h_{I}=h_{L T}\left[1+1.128 x^{0.817}\left(\frac{\rho_{L}}{\rho_{G}}\right)^{0.3685}\left(\frac{\mu_{L}}{\mu_{G}}\right)^{0.2363}\left(1-\frac{\mu_{G}}{\mu_{L}}\right)^{2.144} \operatorname{Pr}_{L}^{-0.1}\right]
$$

For non-circular channels, $\mathrm{D}_{\mathrm{HYD}}$ is to be used as equivalent diameter in Weber and Froude numbers and $\mathrm{D}_{\mathrm{HP}}$ in all other places. $\mathrm{D}_{\mathrm{HP}}$ is defined as:

$$
D_{H P}=\frac{4 x \text { Flow area }}{\text { Perimeter with heat transfer }}
$$

$\mathrm{We}_{\mathrm{GT}}$ is the Weber number assuming all mass to be flowing as vapor, given by:

$$
W e_{G T}=\frac{G^{2} D}{\rho_{G} \sigma}
$$

\subsubsection{Correlations for Flattened Tubes}

A few correlations have been proposed based on data and analysis for flattened tubes. Wen et al. [7] compared the results of their numerical simulation with round tube correlations and the measurements of Kaewon et al. [12] in flattened tubes. On this basis they proposed the following correlation:

$$
h_{T P}=h_{\text {round }} A_{r}^{0.08157}
$$

where $h_{\text {round }}$ is the heat transfer coefficient of a round tube with hydraulic diameter of the flattened tube. They recommended the use of Thome et al. [23] correlation for this purpose.

Based on their own measurements on flattened tubes, Kim et al. [10] proposed the following modified form of Shah [25] correlation.

If it is Regime I of Shah [25] correlation,

$$
h_{T P}=h_{I} A_{r}^{-0.462},
$$

where,

$$
h_{T P}=h_{I} A_{r}^{-0.462}+h_{N u} A_{r}^{0.449}
$$

Solanki and Kumar [13] studied condensation of R-134a in a horizontal $8.91 \mathrm{~mm}$ diameter tube and two channels obtained by flattening it. They correlated their data by the following equation.

$$
h_{T P}=1.635 h_{\text {round }} A_{r}^{0.3163}
$$

They recommend that $\mathrm{h}_{\text {round }}$ be calculated by the Dobson and Chato [22] correlation. All their data were in the annular flow regime.

\section{Data Analysis}

A wide-ranging database was compared to a number of correlations that include general correlations, correlations specifically for mini channels, and correlations for flattened tubes, as described in the following.

\subsection{Data Collection}

A wide-ranging database was available from the author's previous work Shah [3]. It included many data sets for non-circular channels. Further literature search resulted in addition of more data sets. As before, data for oil-containing refrigerants were not considered as oil can significantly affect 
heat transfer. Also, data for mixtures were excluded as their heat transfer is affected by mass transfer effects. Exception was made for R-404a and R-410A as their temperature glide is so small that they behave like pure fluids.

The complete range of data analyzed for channels other than flattened tubes is given in Tables 1 and 2. The range of data for flattened tubes is listed in Table 3.

Table 1. Range of data for non-circular channels other than flattened tubes.

\begin{tabular}{cc}
\hline Parameter & Data Range \\
\hline Fluids & $\begin{array}{c}\text { R-22, R-32, R-134a, R-141b, R-236fa, R-245fa, R-410A, R-152a, R-1234ze(E), } \\
\text { propane, carbon dioxide, FC-72, isobutane, HFE-7100, ammonia (15 fluids) }\end{array}$ \\
\hline Geometry & $\begin{array}{c}\text { square, rectangle, semi-circle, triangle, barrel shaped, N-insert, W-insert, } \\
\text { single and multi channels. }\end{array}$ \\
\hline Orientation & Horizontal, vertical down \\
\hline Aspect Ratio, width/height & 0.14 to 4.0 \\
\hline $\mathrm{D}_{\mathrm{HYD}}, \mathrm{mm}$ & 0.067 to 1.46 \\
\hline Reduced pressure & 0.0449 to 0.7738 \\
\hline $\mathrm{G}, \mathrm{kg} \mathrm{m}{ }^{-2} \mathrm{~s}^{-1}$ & 48 to 1000 \\
\hline $\mathrm{x}, \%$ & 0.01 to 0.99 \\
\hline We & 5 to 4195 \\
\hline Re $_{\mathrm{LT}}$ & 52 to 16,987 \\
\hline Number of data sources & 22 \\
\hline Number of data sets & $42(41$ horizontal, 1 vertical down) \\
\hline
\end{tabular}

\subsection{Correlations Evaluated}

The correlations evaluated include those of Kim and Mudawar [1] for minichannels, Dorao and Fernandino [2], and Shah [3]. These three were included as they were reported to agree with data for many non-circular channels. The correlations of Wen et al. [7], Solanki and Kumar [13], and Kim et al. [10] which are based on data for flattened tubes were also included.

It was intended to compare the data with all general correlations which have been verified with wide-ranging data. However, some of them require heat flux or $\Delta \mathrm{T}$ to be known. Among these are Cavallini et al. [24], Thome et al. [23], and Dobson and Chato [22]. Most published data do not include heat flux or $\Delta \mathrm{T}$. These therefore could not be included for evaluation. Among well-known correlation of the general type those by Akers et al. [36], Ananiev et al. [37], and Moser et al. [38] have been reported to give good agreement by many researchers and do not require heat flux to be known. These were therefore also included in the evaluation. 
Table 2. Deviations of various correlations with data for non-circular channels other than flattened tubes.

\begin{tabular}{|c|c|c|c|c|c|c|c|c|c|c|c|c|c|c|c|c|}
\hline \multirow[b]{2}{*}{ Source } & \multirow[b]{2}{*}{ Channel Type } & \multirow{2}{*}{$\begin{array}{c}\mathrm{D}_{\mathrm{HYD}} \\
\left(\mathrm{D}_{\mathrm{HP}}\right) \\
\mathrm{mm}\end{array}$} & \multirow[b]{2}{*}{ AR } & \multirow[b]{2}{*}{ Fluid } & \multirow[b]{2}{*}{$\mathrm{p}_{\mathrm{r}}$} & \multirow{2}{*}{$\begin{array}{c}\mathrm{G} \\
\mathbf{K g} . \\
\mathbf{m}^{-2} \mathbf{s}^{-1}\end{array}$} & \multirow[b]{2}{*}{$\operatorname{Re}_{\mathrm{LT}}$} & \multirow[b]{2}{*}{$\mathrm{We}_{\mathrm{GT}}$} & \multirow[b]{2}{*}{$\mathbf{N}$} & \multicolumn{7}{|c|}{ Deviation \% Mean Absolute Average } \\
\hline & & & & & & & & & & Shah [3] & $\begin{array}{c}\text { Kim and } \\
\text { Mudawar } \\
\text { [1] }\end{array}$ & $\begin{array}{l}\text { Ananiev } \\
\text { et al. } \\
\text { [37] }\end{array}$ & $\begin{array}{c}\text { Kim et al. } \\
\text { [10] }\end{array}$ & $\begin{array}{c}\text { Solanki } \\
\text { and Kumar } \\
{[13]}\end{array}$ & $\begin{array}{c}\text { Wen et al. } \\
\text { [7] }\end{array}$ & $\begin{array}{c}\text { Dorao and } \\
\text { Fern-andino } \\
{[2]}\end{array}$ \\
\hline \multirow{6}{*}{$\begin{array}{c}\text { Dong and Yang } \\
\text { [39] }\end{array}$} & \multirow{6}{*}{ Multi, rect., $\mathrm{H}$} & \multirow{6}{*}{$\begin{array}{c}0.114 \\
(0.133) \\
0.092 \\
(0.104) \\
0.067 \\
(0.08)\end{array}$} & \multirow{2}{*}{0.4} & \multirow{6}{*}{$\mathrm{R}-141 \mathrm{~b}$} & \multirow{6}{*}{0.0449} & \multirow{2}{*}{200} & \multirow{2}{*}{87} & \multirow{2}{*}{35} & \multirow{2}{*}{20} & 38.4 & 40.4 & 39.9 & 98.0 & 84.8 & 12.7 & 17.7 \\
\hline & & & & & & & & & & 32.5 & 29.7 & 36.2 & 96.0 & 81.3 & 2.1 & 10.1 \\
\hline & & & \multirow{2}{*}{0.3} & & & \multirow{2}{*}{200} & & & & 20.7 & 28.2 & 22.0 & 98.5 & 45.8 & 11.9 & 7.0 \\
\hline & & & & & & & 68 & 28 & 11 & 16.6 & 10.6 & 22.0 & 98.5 & 45.8 & 11.9 & -10.8 \\
\hline & & & 0,5 & & & 200 & 52 & 20 & 12 & 14.9 & 24.1 & 9.6 & 16.2 & 26.2 & 30.9 & 26.9 \\
\hline & & & 0.5 & & & 200 & 52 & 20 & 12 & -14.2 & -14.5 & -9.6 & 15.9 & 26.2 & -30.9 & -26.9 \\
\hline Fronk and & Single, sq. $\mathrm{H}$ & 01 & 1 & $\mathrm{CO}_{2}$ & 0.6871 & 600 & 803 & 114 & 14 & 25.7 & 30.2 & 29.5 & 24.5 & 93.4 & 24.7 & 24.7 \\
\hline Garimella [40] & Single, sq., H & 0.1 & 1 & $\mathrm{CO}_{2}$ & 0.7738 & 600 & 904 & 150 & 14 & -25.7 & -30.2 & -29.5 & -24.5 & 93.4 & -24.7 & -24.7 \\
\hline & & & & & 0.1889 & 600 & 371 & 118 & & 71.2 & 74.1 & 73.7 & 67.6 & 40.5 & 71.6 & 71.6 \\
\hline & & 0.10 & 1 & & 0.4128 & 800 & 646 & 196 & 10 & -71.2 & -74.1 & -73.7 & -67.6 & -40.5 & -71.6 & -71.6 \\
\hline Garimella et al. & & & & & & & & & & 75.4 & 83.4 & 81.1 & 86.8 & 43.1 & 79.0 & 80.8 \\
\hline [41] & Rect., multi, H & 0.15 & 3 & $\mathrm{R}-134 \mathrm{a}$ & 0.1889 & 300 & 245 & 49 & 5 & -75.4 & -83.4 & -81.1 & -86.8 & -43.1 & -79.0 & -80.8 \\
\hline & & 0.16 & & & 0.2494 & 600 & 593 & 188 & & 75.5 & 77.8 & 77.7 & 85.4 & 21.0 & 73.0 & 75.9 \\
\hline & & 0.16 & 4 & & 0.4128 & 800 & 1033 & 313 & 8 & -75.5 & -77.8 & -77.7 & -85.4 & -21.0 & -73.0 & -75.9 \\
\hline & & & & & & 100 & 614 & 32 & & 21.0 & 29.8 & 35.0 & 40.7 & 80.1 & 37.3 & 37.3 \\
\hline & & 0.76 & & & & 400 & 2454 & 519 & 18 & -4.0 & -21.7 & -29.6 & -11.6 & 68.6 & -22.8 & -22.8 \\
\hline Nakashita [42] & Multi, Rect., H & & Un-known & R-134a & 0.4128 & 100 & 856 & 23 & & 11.5 & 23.9 & 28.1 & 29.7 & 83.9 & 29.4 & 29.4 \\
\hline & & 1.06 & & & & 400 & 3421 & 372 & 19 & 3.1 & -15.3 & -16.0 & -4.9 & 79.1 & -18.7 & -18.7 \\
\hline & & & & & 0.210 & 100 & 508 & 27 & & 17.6 & 26.7 & 30.3 & 41.2 & 63.9 & 32.7 & 32.0 \\
\hline & & & & R-1234ze & 0.3499 & 400 & 2598 & 424 & 37 & -6.5 & -21.5 & -26.0 & 6.5 & 48.2 & -30.8 & -28.7 \\
\hline Jige et al [29] & Multi rect $\mathrm{H}$ & & & & 0.4271 & 100 & 893 & 26 & & 20.7 & 32.4 & 32.3 & 38.5 & 96.3 & 33.3 & 33.9 \\
\hline Jige et al. [29] & Multi, rect., $\mathrm{H}$ & 0.85 & 0.69 & R-32 & 0.6780 & 400 & 4752 & 563 & 33 & 4.5 & -3.2 & -15.3 & 7.8 & 90.4 & -11.7 & -18.0 \\
\hline & & & & & 0.2494 & 100 & 524 & 26 & 52 & 21.5 & 27.4 & 35.2 & 31.8 & 55.0 & 34.7 & 34.0 \\
\hline & & & & R-134a & 0.4128 & 400 & 2745 & 445 & 53 & -13.3 & -21.2 & -31.7 & -0.1 & 39.7 & -29.9 & -27.8 \\
\hline Rahman et al. & Multi rect $\mathrm{H}$ & 081 & 05 & R-134a & 02176 & 50 & 235 & 7 & 47 & 25.9 & 37.9 & 52.5 & 29.3 & 28.0 & 54.9 & 52.3 \\
\hline [30] & Multi, rect., $\mathrm{H}$ & 0.81 & 0.5 & R-134a & 0.2176 & 200 & 940 & 111 & 47 & -22.3 & -37.9 & -52.9 & -21.5 & -18.2 & -54.9 & -52.3 \\
\hline & & 0.57 & & & & 48 & 91 & 10 & & 25.9 & 21.2 & 16.0 & 18.5 & 16.2 & 46.9 & 42.8 \\
\hline Al-Zaidi [43] & Multi, rect. H & $(0.67)$ & 0.4 & HFE-7100 & 0.0455 & 126 & 240 & 69 & 36 & 25.9 & -0.5 & -15.2 & 18.0 & 13.9 & -46.9 & -42.8 \\
\hline & & & & & & 200 & 1138 & 124 & & 19.9 & 18.0 & 17.8 & 24.0 & 104.8 & 17.9 & 17.9 \\
\hline & & & & R-1234ze & 0.2733 & 800 & 5136 & 1983 & 18 & -2.7 & -11.3 & -8.5 & 9.3 & 104.8 & -12.3 & -2.3 \\
\hline Lin ot al [44] & Square $\mathrm{H}$ & 0952 & 1 & $P=2$ & & 350 & 2399 & 292 & & 8.6 & 9.7 & 6.9 & 17.2 & 118.2 & 7.7 & 7.7 \\
\hline & & 0.952 & 1 & R-22 & 0.3062 & 500 & 3426 & 526 & 12 & 4.5 & -3.3 & -6.1 & 17.2 & 118.2 & 2.5 & 2.5 \\
\hline & & & & Propane & 0.321 & 200 & 2295 & 238 & & 19.9 & 15.7 & 17.7 & 26.4 & 133.3 & 18.8 & 18.8 \\
\hline & & & & Propane & 0.4017 & 500 & 6416 & 1500 & 26 & 6.2 & -4.7 & -1.5 .5 & 23.1 & 133.3 & 0.7 & 0.7 \\
\hline
\end{tabular}


Table 2. Cont.

\begin{tabular}{|c|c|c|c|c|c|c|c|c|c|c|c|c|c|c|c|c|}
\hline \multirow[b]{2}{*}{ Source } & \multirow[b]{2}{*}{ Channel Type } & \multirow[b]{2}{*}{$\begin{array}{c}D_{\text {HYD }} \\
\left(D_{\text {HP }}\right) \\
m m\end{array}$} & \multirow[b]{2}{*}{ AR } & \multirow[b]{2}{*}{ Fluid } & \multirow[b]{2}{*}{$\mathrm{p}_{\mathrm{r}}$} & \multirow[b]{2}{*}{$\begin{array}{c}\mathrm{G} \\
\mathrm{Kg} . \\
\mathrm{m}^{-2} \mathrm{~s}^{-1}\end{array}$} & \multirow[b]{2}{*}{$\operatorname{Re}_{\mathrm{LT}}$} & \multirow[b]{2}{*}{$\mathrm{We}_{\mathrm{GT}}$} & \multirow[b]{2}{*}{$\mathbf{N}$} & \multicolumn{7}{|c|}{ Deviation \% Mean Absolute Average } \\
\hline & & & & & & & & & & Shah [3] & $\begin{array}{c}\text { Kim and } \\
\text { Mudawar } \\
\text { [1] }\end{array}$ & $\begin{array}{l}\text { Ananiev } \\
\text { et al. } \\
\text { [37] }\end{array}$ & $\begin{array}{c}\text { Kim et al. } \\
\text { [10] }\end{array}$ & $\begin{array}{c}\text { Solanki } \\
\text { and Kumar } \\
{[13]}\end{array}$ & $\begin{array}{l}\text { Wen et al. } \\
{[7]}\end{array}$ & $\begin{array}{c}\text { Dorao and } \\
\text { Fern-andino } \\
{[2]}\end{array}$ \\
\hline \multirow{8}{*}{ Park et al. [45] } & \multirow{6}{*}{ Multi, rect., V } & \multirow{6}{*}{1.43} & \multirow{6}{*}{1.86} & \multirow{2}{*}{ R-1234ze } & 0.2100 & 100 & 855 & 51 & \multirow[b]{2}{*}{31} & 29.9 & 20.7 & 21.0 & 18.9 & 187.3 & 24.1 & 21.7 \\
\hline & & & & & 0.4417 & 260 & 2137 & 317 & & 22.3 & 19.3 & 8.5 & -4.5 & 187.3 & 8.0 & 2.7 \\
\hline & & & & \multirow{2}{*}{$\mathrm{R}-236 \mathrm{fa}$} & & 100 & 604 & 63 & \multirow{2}{*}{17} & 40.3 & 23.4 & 23.8 & 15.0 & 176.8 & 30.0 & 15.0 \\
\hline & & & & & 0.1359 & 260 & 1571 & 423 & & 31.9 & 23.2 & 17.9 & -2.7 & 176.8 & 20.4 & -2.7 \\
\hline & & & & \multirow{4}{*}{$\mathrm{R}-134 \mathrm{a}$} & & 100 & 884 & 47 & & 12.8 & 8.2 & 12.6 & 15.9 & 149.3 & 16.0 & 14.4 \\
\hline & & & & & 0.2494 & 260 & 2295 & 316 & 16 & 7.4 & 3.5 & -6.3 & -15.5 & 149.3 & 2.8 & -2.9 \\
\hline & Multi, Square, & & & & & 150 & 861 & 52 & & 25.2 & 13.3 & 19.5 & 40.4 & 159.8 & 24.4 & 24.4 \\
\hline & $\mathrm{H}$ & 1 & 0.762 & & 0.166 & 750 & 4303 & 1307 & 31 & 25.1 & 10.0 & 11.7 & 39.9 & 159.8 & 21.4 & 21.4 \\
\hline & Multi, & & & & & 150 & 827 & 50 & & 12.2 & 20.8 & 21.7 & 14.5 & 90.8 & 14.9 & 14.9 \\
\hline & W-insert, $\mathrm{H}$ & 1 & 0.732 & & 0.366 & 750 & 4134 & 1256 & 25 & -6.3 & -18.9 & -17.6 & 3.1 & 90.8 & -10.6 & -10.6 \\
\hline & Multi, N- & & & & & 300 & 1211 & 147 & 16 & 22.3 & 27.8 & 28.1 & 18.9 & 77.2 & 22.7 & 22.7 \\
\hline Agarwal et al. & shape, $\mathrm{H}$ & 1 & 0.536 & R-134a & 0.3661 & 750 & 3017 & 919 & 16 & -15.2 & -25.8 & -22.9 & -3.9 & 77.2 & -16.7 & -16.7 \\
\hline & Multi, triangle, & 1 & & & & 150 & 948 & 71 & 15 & 14.0 & 24.7 & 27.1 & 45.6 & 193.1 & 30.4 & 25.8 \\
\hline & $\mathrm{H}$ & 1 & 0.839 & & 0.3661 & 750 & 16,987 & 2042 & 15 & -8.7 & -23.2 & -26.4 & -32.5 & 193.1 & -30.2 & -23.8 \\
\hline & Multi, barrel, & & & & & 150 & 1805 & 55 & & 29.2 & 24.8 & 31.0 & 31.1 & 150.5 & 29.5 & 29.0 \\
\hline & $\mathrm{H}$ & 0.799 & 1.25 & & 0.3661 & 750 & 4512 & 1370 & 150750 & 12.7 & -2.4 & 2.0 & 14.1 & 150.5 & 11.2 & 9.2 \\
\hline & & & & & & 600 & 1437 & 262 & & 24.5 & 26.8 & 27.6 & 29.0 & 140.9 & 23.4 & 24.3 \\
\hline & Multi, rect., H & 0.424 & 2 & & 0.3661 & 750 & 2394 & 727 & 10 & -7.8 & -22.2 & -15.2 & -23.4 & 140.9 & -2.9 & -8.3 \\
\hline & & & & & 0.2529 & 175 & 2129 & 224 & & 21.5 & 18.8 & 16.1 & 33.3 & 152.1 & 16.5 & 16.5 \\
\hline Belchi et al. [47] & Multt, sq., $\mathrm{H}$ & 1.16 & 1 & Propane & 0.4017 & 350 & 5472 & 896 & 28 & 16.6 & 10.1 & 7.1 & 33.1 & 152.1 & 7.9 & 7.9 \\
\hline & & & & & & 100 & 305 & 16 & & 19.0 & 25.6 & 30.4 & 37.1 & 75.2 & 31.4 & 31.4 \\
\hline Shin and Kim & Single, square, & 0.494 & 1 & R-134a & 0.2494 & 600 & 1832 & 581 & 11 & -3.6 & -20.4 & -24.6 & -9.1 & 66.2 & -21.8 & -21.8 \\
\hline [48] & $\mathrm{H}$ & & & $\mathrm{K}-134 \mathrm{a}$ & 0.2494 & 100 & 601 & 32 & & 28.0 & 25.8 & 28.6 & 37.4 & 110.6 & 30.7 & 30.7 \\
\hline & & 0.972 & 1 & & & 600 & 3605 & 1144 & 23 & 7.8 & -2.5 & -7.2 & 12.0 & 106.8 & -3.6 & -3.6 \\
\hline Liu et al. [49] & Single, square, & & & & & 200 & 1386 & 174 & & 15.9 & 13.9 & 13.1 & 18.6 & 115.4 & 14.9 & 14.9 \\
\hline Liu et al. [49] & $\mathrm{H}$ & 0.952 & 1 & $\mathrm{R}-1 \mathrm{bza}$ & 0.2005 & 600 & 4157 & 1567 & 21 & 3.6 & -0.8 & -2.1 & 11.3 & 115.4 & -9.5 & -9.5 \\
\hline Del Col et al. & Single, square, & 1.23 & & R-134a & 0.2494 & 200 & 1521 & 161 & & 14.8 & 21.3 & 22.9 & 8.8 & 70.8 & 20.4 & 20.4 \\
\hline [14] & $\mathrm{H}$ & 1.23 & 1 & $\mathrm{R}-134 \mathrm{a}$ & 0.2494 & 789 & 6000 & 2503 & 44 & -14.8 & -21.3 & -22.9 & -6.7 & 70.1 & -20.4 & -20.4 \\
\hline & Single, square, & & & $R-32$ & & 100 & 1292 & 37 & & 12.0 & 23.3 & 32.9 & 23.5 & 66.3 & 27.9 & 27.9 \\
\hline Del Col et al. & & 1.23 & 1 & K-32 & $0.42 / 1$ & 390 & 5041 & 568 & 30 & -11.9 & -23.3 & -32.9 & -22.1 & 66.3 & -27.9 & -27.9 \\
\hline [50] & Single, square, & 1.23 & & R-134a & 0.2494 & 100 & 760 & 90 & 53 & 10.9 & 28.7 & 37.0 & 24.2 & 40.2 & 34.1 & 34.1 \\
\hline & $\mathrm{V}$ & 1.23 & 1 & R-134a & 0.2494 & 390 & 2966 & 610 & 53 & -10.7 & -28.7 & -37.0 & -23.8 & 40.2 & -34.1 & -34.1 \\
\hline Kim \& & Multi, square, & 1.0 & 1 & FC-72 & 00574 & 68 & 141 & 32 & 54 & 20.4 & 28.4 & 34.3 & 30.9 & 29.6 & 24.6 & 24.6 \\
\hline Mudawar. [20] & $\mathrm{H}$ & $(1.33)$ & 1 & $\mathrm{FC}-12$ & $0.05 / 4$ & 367 & 763 & 932 & 54 & -7.2 & -17.0 & -34.3 & -30.7 & 22.2 & -15.7 & -15.7 \\
\hline & Multi, square, & 1.0 & & & 0.2176 & 75 & 579 & 18 & 61 & 10.9 & 14.2 & 23.0 & 20.4 & 66.7 & 19.3 & 19.9 \\
\hline & & $(1.33)$ & 1 & & 0.2846 & 450 & 3946 & 693 & 61 & 3.1 & 5.3 & -21.9 & -2.8 & 66.7 & -15.4 & -15.4 \\
\hline & Multi, & 1.0 & 2 & R-134a & 0.2176 & 75 & 714 & 19 & 31 & 5.4 & 10.4 & 26.0 & 33.0 & 93.1 & 20.5 & 21.4 \\
\hline Derby et al. [51] & semi-circle, $\mathrm{H}$ & $(1.64)$ & 2 & N-104a & 0.2176 & 450 & 4282 & 693 & 31 & -2.6 & 5.1 & -26.1 & -30.0 & 93.1 & -13.3 & -18.0 \\
\hline & Multi, triang., & 1.0 & 1.16 & & & 75 & 653 & 19 & 25 & 9.8 & 16.6 & 25.3 & 22.9 & 65.4 & 24.1 & 24.1 \\
\hline & $\mathrm{H}$ & (1.5) & 1.16 & & 0.2176 & 450 & 3917 & 693 & 25 & -2.5 & 3.6 & -25.3 & -14.0 & 65.4 & -16.5 & -17.5 \\
\hline
\end{tabular}


Table 2. Cont.

\begin{tabular}{|c|c|c|c|c|c|c|c|c|c|c|c|c|c|c|c|c|}
\hline \multirow[b]{2}{*}{ Source } & \multirow[b]{2}{*}{ Channel Type } & \multirow[b]{2}{*}{$\begin{array}{c}D_{\text {HYD }} \\
\left(D_{\text {HP }}\right) \\
m m\end{array}$} & \multirow[b]{2}{*}{ AR } & \multirow[b]{2}{*}{ Fluid } & \multirow[b]{2}{*}{$\mathrm{p}_{\mathrm{r}}$} & \multirow[b]{2}{*}{$\begin{array}{c}\mathrm{G} \\
\mathrm{Kg} . \\
\mathrm{m}^{-2} \mathbf{s}^{-1}\end{array}$} & \multirow[b]{2}{*}{$\operatorname{Re}_{\mathrm{LT}}$} & \multirow[b]{2}{*}{$\mathrm{We}_{\mathrm{GT}}$} & \multirow[b]{2}{*}{$\mathbf{N}$} & \multicolumn{7}{|c|}{ Deviation \% Mean Absolute Average } \\
\hline & & & & & & & & & & Shah [3] & $\begin{array}{c}\text { Kim and } \\
\text { Mudawar } \\
\text { [1] }\end{array}$ & $\begin{array}{l}\text { Ananiev } \\
\text { et al. } \\
\text { [37] }\end{array}$ & $\begin{array}{c}\text { Kim et al. } \\
{[10]}\end{array}$ & $\begin{array}{c}\text { Solanki } \\
\text { and Kumar } \\
{[13]}\end{array}$ & $\begin{array}{c}\text { Wen et al. } \\
\text { [7] }\end{array}$ & $\begin{array}{c}\text { Dorao and } \\
\text { Fern-andino } \\
{[2]}\end{array}$ \\
\hline $\begin{array}{c}\text { Cavallini et al. } \\
\text { [52] }\end{array}$ & Multi, sq., H & 1.4 & 1 & $\mathrm{R}-410 \mathrm{~A}$ & 0.4917 & $\begin{array}{c}200 \\
1000\end{array}$ & $\begin{array}{c}2906 \\
14,531\end{array}$ & $\begin{array}{r}168 \\
4195\end{array}$ & 27 & $\begin{array}{l}11.1 \\
-5.8\end{array}$ & $\begin{array}{c}18.8 \\
-17.9\end{array}$ & $\begin{array}{c}14.7 \\
-12.0\end{array}$ & $\begin{array}{c}12.1 \\
4.2\end{array}$ & $\begin{array}{l}119.9 \\
119.9\end{array}$ & $\begin{array}{l}10.8 \\
-6.5\end{array}$ & $\begin{array}{l}10.8 \\
-6.5 \\
\end{array}$ \\
\hline $\begin{array}{c}\text { Koyama et al. } \\
{[53]}\end{array}$ & Multi, rect, $\mathrm{H}$ & 0.807 & 0.3 & $\mathrm{R}-134 \mathrm{a}$ & 0.4177 & $\begin{array}{l}273 \\
652 \\
\end{array}$ & $\begin{array}{l}1791 \\
4278 \\
\end{array}$ & $\begin{array}{c}184 \\
1052 \\
\end{array}$ & 8 & $\begin{array}{c}11.1 \\
2.5 \\
\end{array}$ & $\begin{array}{l}26.1 \\
10.8 \\
\end{array}$ & $\begin{array}{c}12.0 \\
-11.4 \\
\end{array}$ & $\begin{array}{l}90.8 \\
90.8 \\
\end{array}$ & $\begin{array}{l}49.3 \\
49.3 \\
\end{array}$ & $\begin{array}{c}12.5 \\
-11.7 \\
\end{array}$ & $\begin{array}{l}10.9 \\
-2.6 \\
\end{array}$ \\
\hline $\begin{array}{l}\text { Al-Hajiri et al. } \\
{[54]}\end{array}$ & Single, rect., $\mathrm{H}$ & 0.7 & 0.14 & $\mathrm{R}-245 \mathrm{fa}$ & $\begin{array}{l}0.1889 \\
0.5197 \\
0.0484 \\
0.1663\end{array}$ & $\begin{array}{c}50 \\
500 \\
50 \\
500\end{array}$ & $\begin{array}{c}246 \\
2464 \\
121 \\
1208 \\
\end{array}$ & $\begin{array}{c}5 \\
539 \\
9 \\
872 \\
\end{array}$ & 14 & $\begin{array}{l}22.6 \\
-1.1 \\
14.5 \\
-4.2 \\
\end{array}$ & $\begin{array}{c}26.3 \\
-18.7 \\
20.8 \\
-15.4 \\
\end{array}$ & $\begin{array}{c}29.0 \\
-28.8 \\
19.7 \\
-14.2 \\
\end{array}$ & $\begin{array}{l}121.0 \\
121.0 \\
127.0 \\
127.0\end{array}$ & $\begin{array}{c}18.7 \\
-10.3 \\
20.4 \\
-10.9 \\
\end{array}$ & $\begin{array}{c}33.1 \\
-33.1 \\
30.1 \\
-27.6 \\
\end{array}$ & $\begin{array}{c}24.2 \\
-21.5 \\
22.9 \\
-15.0\end{array}$ \\
\hline Wang et al. [55] & Multi, rect., $\mathrm{H}$ & 1.46 & 1.07 & $\mathrm{R}-134 \mathrm{a}$ & 0.4586 & $\begin{array}{l}150 \\
750 \\
\end{array}$ & $\begin{array}{l}1857 \\
9434 \\
\end{array}$ & $\begin{array}{c}102 \\
2561 \\
\end{array}$ & 37 & $\begin{array}{c}13.0 \\
6.8 \\
\end{array}$ & $\begin{array}{l}10.8 \\
-5.0 \\
\end{array}$ & $\begin{array}{l}12.9 \\
-4.4 \\
\end{array}$ & $\begin{array}{l}17.8 \\
15.2 \\
\end{array}$ & $\begin{array}{l}128.2 \\
128.2 \\
\end{array}$ & $\begin{array}{c}11.8 \\
3.6 \\
\end{array}$ & $\begin{array}{c}11.6 \\
3.0 \\
\end{array}$ \\
\hline Kim et al. [10] & Multi, rect., $\mathrm{H}$ & 1.4 & 1.4 & $\mathrm{R}-410 \mathrm{~A}$ & 0.3453 & $\begin{array}{l}200 \\
600 \\
200 \\
600\end{array}$ & $\begin{array}{l}3162 \\
9482 \\
2154 \\
6461\end{array}$ & $\begin{array}{c}177 \\
1618 \\
139 \\
1254\end{array}$ & 10 & $\begin{array}{l}12.0 \\
11.8 \\
21.8 \\
21.8\end{array}$ & $\begin{array}{l}8.1 \\
2.6 \\
9.0 \\
8.9\end{array}$ & $\begin{array}{c}8.2 \\
3.7 \\
12.9 \\
8.4\end{array}$ & $\begin{array}{c}6.5 \\
3.1 \\
18.1 \\
17.0\end{array}$ & $\begin{array}{l}196.7 \\
196.7 \\
187.0 \\
187.0\end{array}$ & $\begin{array}{l}14.9 \\
14.9 \\
23.7 \\
23.7\end{array}$ & $\begin{array}{l}11.8 \\
11.8 \\
20.6 \\
20.6\end{array}$ \\
\hline All sources & & $\begin{array}{l}0.067 \\
1.46\end{array}$ & $\begin{array}{c}0.14 \\
4.0\end{array}$ & & $\begin{array}{l}0.0449 \\
0.7738\end{array}$ & $\begin{array}{c}48 \\
1000\end{array}$ & $\begin{array}{c}52 \\
16,987\end{array}$ & $\begin{array}{c}5 \\
4195\end{array}$ & 1120 & $\begin{array}{l}20.3 \\
-1.8\end{array}$ & $\begin{array}{c}24.0 \\
-11.3\end{array}$ & $\begin{array}{c}27.4 \\
-18.4\end{array}$ & $\begin{array}{c}31.4 \\
2.6\end{array}$ & $\begin{array}{l}84.6 \\
78.0\end{array}$ & $\begin{array}{c}27.2 \\
-16.5\end{array}$ & $\begin{array}{c}26.5 \\
-16.0\end{array}$ \\
\hline
\end{tabular}

Table 3. Deviations of various correlations with data for flattened tubes and the round tubes from which they were made.

\begin{tabular}{|c|c|c|c|c|c|c|c|c|c|c|c|c|c|c|c|c|}
\hline \multirow[b]{2}{*}{ Source } & \multirow[b]{2}{*}{ Geometry } & \multirow[b]{2}{*}{$\begin{array}{c}D_{H Y D} \\
\left(D_{H P}\right) \\
m m\end{array}$} & \multirow[b]{2}{*}{ AR } & \multirow[b]{2}{*}{ Fluid } & \multirow[b]{2}{*}{$\mathrm{p}_{\mathrm{r}}$} & \multirow[b]{2}{*}{$\begin{array}{c}\mathrm{G} \\
\mathrm{Kg} . \\
\mathrm{m}^{-2} \mathbf{s}^{-1}\end{array}$} & \multirow[b]{2}{*}{$\operatorname{Re}_{\mathrm{LT}}$} & \multirow[b]{2}{*}{ We $_{\mathrm{GT}}$} & \multirow[b]{2}{*}{$\mathbf{N}$} & \multicolumn{7}{|c|}{ Deviation, \% Mean Absolute Average } \\
\hline & & & & & & & & & & Shah [3] & $\begin{array}{c}\text { Kim and } \\
\text { Mudawar } \\
\text { [1] }\end{array}$ & $\begin{array}{l}\text { Ana-niev } \\
\text { et al. } \\
\text { [37] }\end{array}$ & $\begin{array}{c}\text { Kim et al. } \\
\text { [10] }\end{array}$ & $\begin{array}{c}\text { Solanki } \\
\text { and Kumar } \\
{[13]}\end{array}$ & $\begin{array}{c}\text { Wen et al. } \\
\text { [7] }\end{array}$ & $\begin{array}{c}\text { Dorao and } \\
\text { Fernandino } \\
\text { [2] }\end{array}$ \\
\hline \multirow{8}{*}{$\begin{array}{l}\text { Wilson et al. } \\
\text { [9] }\end{array}$} & \multirow{4}{*}{$\begin{array}{c}\text { Flattened } \\
\text { tube, } \mathrm{H}\end{array}$} & \multirow{4}{*}{4.88} & \multirow{4}{*}{4.4} & \multirow{2}{*}{$\mathrm{R}-134 \mathrm{a}$} & \multirow[b]{2}{*}{0.2176} & 75 & 1915 & 85 & \multirow{2}{*}{15} & 13.5 & 27.1 & 27.6 & 46.5 & 152.4 & 20.7 & 27.3 \\
\hline & & & & & & 400 & 10,213 & 2409 & & -4.7 & -24.8 & -27.6 & -38.0 & 152.4 & -3.0 & -27.3 \\
\hline & & & & \multirow{2}{*}{ R-410A } & \multirow{2}{*}{0.4347} & 75 & 3191 & 71 & \multirow{2}{*}{15} & 12.8 & 24.7 & 27.1 & 45.6 & 193.1 & 17.3 & 25.8 \\
\hline & & & & & & 400 & 16,987 & 2042 & & -0.7 & -23.2 & -26.4 & -32.5 & 193.1 & -17.3 & -23.6 \\
\hline & \multirow{4}{*}{$\begin{array}{l}\text { Round } \\
\text { tube, } \mathrm{H}\end{array}$} & \multirow{4}{*}{8.9} & \multirow{4}{*}{1} & \multirow{2}{*}{$\mathrm{R}-134 \mathrm{a}$} & \multirow{2}{*}{0.2176} & 75 & 3873 & 171 & \multirow{2}{*}{7} & 43.1 & 37.8 & 26.9 & 43.1 & 82.8 & 29.0 & 29.0 \\
\hline & & & & & & 175 & 9038 & 933 & & 43.1 & 27.5 & -12.6 & 43.1 & 72.0 & -14.0 & -14.0 \\
\hline & & & & \multirow{2}{*}{ R-410A } & \multirow{2}{*}{0.4347} & 75 & 6455 & 143 & \multirow{2}{*}{15} & 54.7 & 19.3 & 25.9 & 54.7 & 128.1 & 28.4 & 28.4 \\
\hline & & & & & & 175 & 15,038 & 791 & & 54.7 & 10.9 & -4.5 & 54.7 & 127.4 & -1.4 & -1.4 \\
\hline
\end{tabular}


Table 3. Cont

\begin{tabular}{|c|c|c|c|c|c|c|c|c|c|c|c|c|c|c|c|c|}
\hline \multirow[b]{2}{*}{ Source } & \multirow[b]{2}{*}{ Geometry } & \multirow[b]{2}{*}{$\begin{array}{c}D_{H Y D} \\
\left(D_{H P}\right) \\
m m\end{array}$} & \multirow[b]{2}{*}{ AR } & \multirow[b]{2}{*}{ Fluid } & \multirow[b]{2}{*}{$\mathrm{p}_{\mathrm{r}}$} & \multirow[b]{2}{*}{$\begin{array}{c}\mathrm{G} \\
\mathrm{Kg} . \\
\mathrm{m}^{-2} \mathbf{s}^{-1}\end{array}$} & \multirow[b]{2}{*}{$\operatorname{Re}_{\mathrm{LT}}$} & \multirow[b]{2}{*}{$\mathrm{We}_{\mathrm{GT}}$} & \multirow[b]{2}{*}{$\mathbf{N}$} & \multicolumn{7}{|c|}{ Deviation, \% Mean Absolute Average } \\
\hline & & & & & & & & & & Shah [3] & $\begin{array}{c}\text { Kim and } \\
\text { Mudawar } \\
\text { [1] }\end{array}$ & $\begin{array}{l}\text { Ana-niev } \\
\text { et al. } \\
\text { [37] }\end{array}$ & $\underset{[10]}{\text { Kim et al. }}$ & $\begin{array}{c}\text { Solanki } \\
\text { and Kumar } \\
{[13]}\end{array}$ & $\begin{array}{c}\text { Wen et al. } \\
\text { [7] }\end{array}$ & $\begin{array}{c}\text { Dorao and } \\
\text { Fernandino } \\
\text { [2] }\end{array}$ \\
\hline \multirow{6}{*}{$\begin{array}{c}\text { Kim et al. } \\
\text { [10] }\end{array}$} & \multirow{5}{*}{$\begin{array}{c}\text { Flattened } \\
\text { tube, } \mathrm{H}\end{array}$} & & 6 & \multirow{6}{*}{ R-410a } & 0.5542 & 100 & 2579 & 72 & 10 & 58.9 & 30.2 & 24.0 & 39.1 & 434.4 & 47.8 & 28.9 \\
\hline & & \multirow{2}{*}{$\begin{array}{l}2.3 \\
3.0\end{array}$} & & & \multirow{2}{*}{0.5542} & 100 & 3364 & 94 & \multirow{2}{*}{10} & 50.7 & 21.5 & 28.8 & 36.1 & 392.6 & 50.8 & 34.6 \\
\hline & & & 4 & & & 400 & 13,451 & 1529 & & 50.7 & 21.5 & 28.8 & 4.7 & 392.6 & 50.8 & 34.6 \\
\hline & & \multirow{2}{*}{4.1} & \multirow{2}{*}{2} & & \multirow{2}{*}{0.5542} & 100 & 4597 & 129 & \multirow{2}{*}{12} & 32.2 & 6.4 & 8.9 & 30.6 & 218.2 & 15.0 & 9.4 \\
\hline & & & & & & 400 & 18,383 & 2018 & & 32.2 & 0.5 & 4.9 & 15.7 & 218.2 & 15.0 & 8.6 \\
\hline & Round $\mathrm{H}$ & 50 & & & 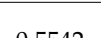 & 100 & 5606 & 157 & & 29.1 & 16.0 & 18.2 & 29.1 & 164.1 & 21.0 & 21.0 \\
\hline & & & & & & 450 & 10,001 & 2654 & & 41.0 & 54.1 & 44.7 & 71.4 & 105.7 & 35.7 & 44.4 \\
\hline & Flattened & 3.8 & 5.88 & & $0.21 / 6$ & 650 & 14,446 & 5536 & 9 & -41.0 & -54.1 & -44.7 & -71.4 & 105.7 & -35.7 & -44.4 \\
\hline $\begin{array}{l}\text { Solanki and } \\
\text { Kumar }\end{array}$ & Tube, $\mathrm{H}$ & & & R-134a & & 450 & 16,790 & 4455 & 9 & 24.5 & 43.6 & 29.3 & 47.7 & 106.3 & 24.0 & 28.8 \\
\hline Kumar & & 6.4 & 2.72 & R-134a & 0.2176 & 650 & 24,253 & 9295 & 9 & -24.5 & -43.6 & -29.3 & -47.7 & 106.3 & -22.7 & -28.8 \\
\hline & Round, $\mathrm{H}$ & 8.9 & 1 & & 0.2176 & 550 & 23,236 & 6173 & & 10.7 & 34.5 & 17.3 & 10.6 & 78.9 & 15.5 & 15.5 \\
\hline & & 0.9 & 1 & & 0.2846 & 650 & 33,607 & 12,880 & 19 & -0.6 & -34.5 & -5.6 & -0.6 & 78.9 & -15.0 & -15.0 \\
\hline & & 8.2 & 1.5 & & 0.1536 & & 10,010 & 1686 & & 20.6 & 35.9 & 23.3 & 26.5 & 81.5 & 26.3 & 28.7 \\
\hline & & 0.2 & 1.5 & & 0.1536 & 155 & 10,010 & 1686 & 7 & -20.6 & -35.9 & -23.3 & -26.5 & 81.5 & -26.3 & -28.7 \\
\hline & Flattened & 729 & 206 & & 01536 & 155 & 8899 & 1499 & 28 & 29.5 & 49.2 & 38.2 & 49.5 & 56.9 & 39.6 & 43.1 \\
\hline & & 5.1 & 3.84 & & 0.1536 & $15 b$ & 6226 & 1049 & 7 & -42.0 & -54.1 & -49.6 & -68.8 & 59.6 & -47.8 & -53.2 \\
\hline & Round, $\mathrm{H}$ & 8.7 & 1 & & 0.142 & 155 & 10,459 & 1746 & & 7.7 & 30.8 & 14.6 & 7.7 & 73.6 & -37.9 & -37.9 \\
\hline & Kouma, 11 & 8.7 & 1 & & 0.1617 & 265 & 18,798 & 5022 & 28 & -1.9 & -30.8 & -14.2 & -1.9 & 73.6 & -32.6 & -32.6 \\
\hline & Single, & & & & 0.1959 & 380 & 7380 & 1807 & & 48.8 & 15.8 & 28.8 & 48.8 & 168.6 & 28.9 & 28.9 \\
\hline & Round, $\mathrm{H}$ & 3.51 & 1 & & 0.2944 & 750 & 16,150 & 6486 & 31 & 48.3 & 9.6 & 25.8 & 48.3 & 168.6 & 27.0 & 27.0 \\
\hline & & & & & & 400 & 7779 & 1666 & & 12.1 & 19.0 & 15.3 & 37.6 & 86.1 & 12.6 & 12.4 \\
\hline Kaewon et & & 3.17 & 0.72 & R-134a & 0.2454 & 825 & 16,045 & 7088 & 11 & 3.9 & -15.1 & 1.5 & 37.6 & 86.1 & -2.7 & 0.0 \\
\hline & Flattened, & 1.84 & 35 & & 02454 & 400 & 4515 & 967 & & 15.3 & 25.7 & 18.9 & 38.7 & 187.2 & 12.7 & 15.0 \\
\hline & $\mathrm{H}$ & 1.84 & 3.5 & & 0.2454 & 825 & 9313 & 4114 & 17 & -3.2 & -17.8 & -7.3 & -38.7 & 187.2 & 3.2 & -6.8 \\
\hline & & 1.16 & 7 & & 0.2454 & 400 & 2847 & 610 & & 44.6 & 48.0 & 46.1 & 74.0 & 109.6 & 39.7 & 46.5 \\
\hline & & & & & 0.2454 & 825 & 5871 & 2594 & 18 & 42.8 & -47.3 & -45.7 & -74.0 & 109.6 & -35.9 & -45.3 \\
\hline & & 1.16 & 0.72 & & 0.1420 & 75 & 1915 & 71 & & 29.6 & 30.8 & 26.7 & 40.5 & 142.8 & 27.3 & 28.2 \\
\hline All & flattened & 8.9 & 7.0 & & 0.5542 & 825 & 33,607 & 12,880 & 271 & 8.2 & -20.3 & -12.6 & -12.7 & 142.6 & -7.7 & -12.7 \\
\hline
\end{tabular}




\subsection{Calculation Methodology}

For the Shah correlation, $\mathrm{D}_{\mathrm{HP}}$ was used except that $\mathrm{D}_{\mathrm{HYD}}$ was used in calculating Weber number and Froude number. The same was also done for other correlations except those of Kim and Mudawar and Dorao and Fernandino for which $\mathrm{D}_{\mathrm{HYD}}$ was used in all its equations as this was specified in them. It is to be noted that for channels cooled on all sides, $\mathrm{D}_{\mathrm{HYD}}=\mathrm{D}_{\mathrm{HP}}$.

Wen et al. [7] correlation recommends the use of Thome et al. [23] correlation for calculating $h_{\text {round }}$. That correlation is flow pattern based. There is no well-verified method to determine flow patterns in mini channels or non-circular channels. It was therefore decided to instead use the correlation of Dorao and Fernadino [2] for this purpose which has been verified with a wide range of data for round tubes and does not require flow pattern determination.

Properties of FC-72 and HFE-7100 were obtained from their manufacturer, 3-M Corporation. For all other fluids REFPROP 9.1 was used. All properties were calculated at the saturation temperature.

\subsection{Results of Data Analysis}

The results of data analysis for flattened tubes are given in Table 3 and for all other non-circular channels in Table 2. The correlations of Akers et al. and Moser et al. gave large deviations; their results are not shown in Tables 2 and 3 but are included in Table 4 . In these tables,

Table 4. Effect of Weber number on the accuracy of various correlations for data of non-circular channels other than flattened tubes.

\begin{tabular}{|c|c|c|c|c|c|c|c|c|c|c|}
\hline \multirow[b]{2}{*}{$\mathrm{We}_{\mathrm{GT}}$} & \multirow[b]{2}{*}{$\mathbf{N}$} & \multicolumn{9}{|c|}{ Deviation \% Mean Absolute Average } \\
\hline & & $\begin{array}{c}\text { Shah } \\
{[3]}\end{array}$ & $\begin{array}{l}\text { Kim and } \\
\text { Mudawar } \\
\text { [1] }\end{array}$ & $\begin{array}{l}\text { Ananiev } \\
\text { et al. } \\
\text { [37] }\end{array}$ & $\begin{array}{l}\text { Kim } \\
\text { et al. } \\
{[10]}\end{array}$ & $\begin{array}{l}\text { Solanki } \\
\text { and } \\
\text { Kumar }\end{array}$ & $\begin{array}{c}\text { Wen } \\
\text { et al. } \\
{[7]}\end{array}$ & $\begin{array}{c}\text { Dorao and } \\
\text { Fern-andino } \\
{[2]}\end{array}$ & $\begin{array}{c}\text { Moser } \\
\text { et al. } \\
{[38]}\end{array}$ & $\begin{array}{c}\text { Akers } \\
\text { et al. } \\
{[38]}\end{array}$ \\
\hline \multirow{2}{*}{$\leq 100$} & \multirow{2}{*}{381} & 21.6 & 32.6 & 41.1 & 40.5 & 36.0 & 40.8 & 40.0 & 39.3 & 191.2 \\
\hline & & -1.6 & -19.5 & -35.1 & -11.5 & -21.6 & -38.7 & -37.2 & 8.3 & 190.6 \\
\hline \multirow{2}{*}{$>100$} & \multirow{2}{*}{739} & 19.7 & 19.5 & 20.3 & 26.7 & 109.7 & 20.1 & 19.5 & 37.4 & 129.7 \\
\hline & & -1.9 & -7.1 & -9.7 & 10.1 & 107.1 & -5.1 & -5.2 & 30.6 & 129.0 \\
\hline \multirow{2}{*}{ All } & \multirow{2}{*}{1120} & 20.3 & 24.0 & 27.4 & 31.4 & 84.6 & 27.2 & 26.5 & 38.1 & 150.7 \\
\hline & & -1.8 & -11.3 & -18.4 & 2.6 & 78.0 & -16.5 & -16.0 & 23.0 & 150.0 \\
\hline
\end{tabular}

Mean absolute deviation $(\mathrm{MAD}) \delta_{\mathrm{m}}$ is defined as:

$$
\delta_{m}=\frac{1}{N} \sum_{1}^{N} A B S\left\{\left(h_{\text {predicted }}-h_{\text {measured }}\right) / h_{\text {measured }}\right\}
$$

Average deviation is defined as:

$$
\delta_{\text {avg }}=\frac{1}{N} \sum_{1}^{\mathrm{N}}\left\{\left(\mathrm{h}_{\text {predicted }}-\mathrm{h}_{\text {measured }}\right) / \mathrm{h}_{\text {measured }}\right\}
$$

It is seen in Table 2 that almost all data sets for channels other than flattened tubes give good agreement with the Shah [3] correlation, the MAD for the 1120 data points from 22 sources being 20.3\%. The correlations of Kim and Mudawar [1] and Dorao and Fernandino [2] also give fairly good agreement with MAD of 24.0 and 26.5 percent, respectively. As seen in Table 3, the results with the flattened tube data are not good for any of the correlations.

\section{Discussion}

\subsection{Channels Other Than Flattened Tubes}

Table 2 gives the results of comparison of data for non-circular channels except flattened tubes with various correlations. It is seen that the best agreement is with the Shah [3] correlation. The only 
data set which gives very large MAD is that of Garimella et al. [41] in rectangular channels. These data are very high compared to all correlations. Figure 1 shows the MAD of Shah correlation for all data sets vs channel hydraulic diameter. Three data points have MAD about $70 \%$, much larger than all other data. These are from Garimella et al. [41]. It is seen that many other data points in the same diameter range and even smaller diameters are in satisfactory agreement. Results over the extreme limits of data do not show any increase in deviation.

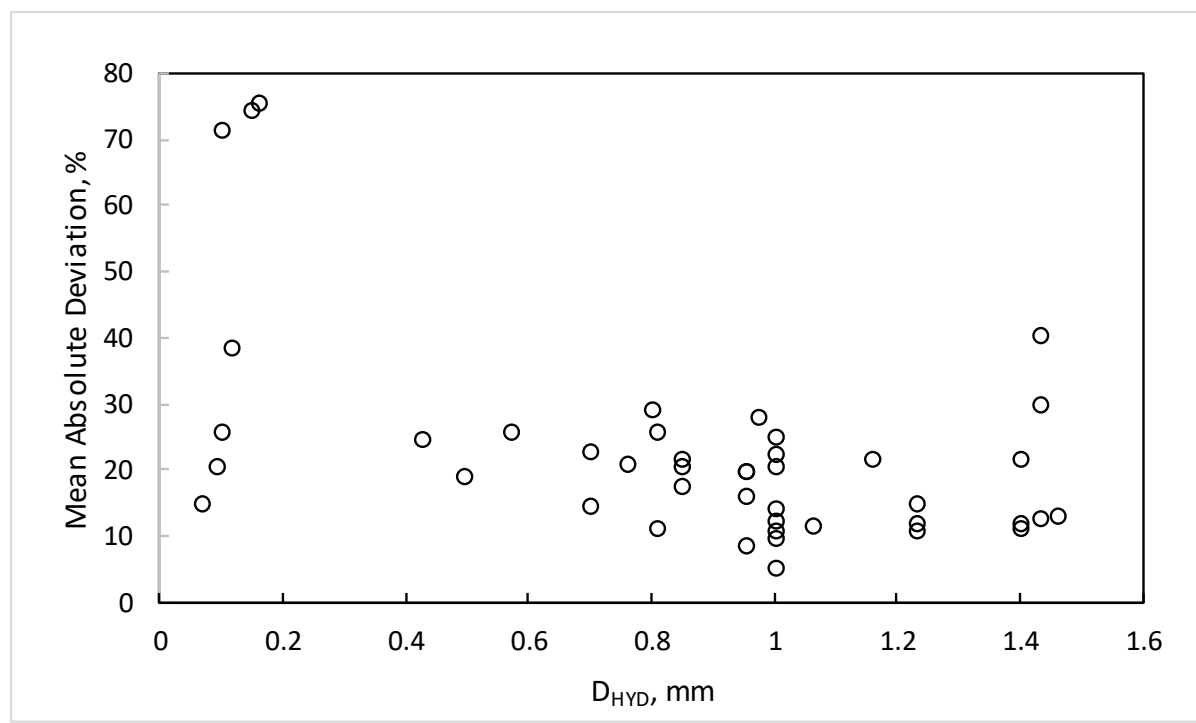

Figure 1. Mean absolute deviations of data sets with Shah [3] correlation vs hydraulic diameter for channels other than flattened tubes.

Figure 2 shows the MAD of the Shah correlation with all data sets as a function of aspect ratio of channel. Again, three data points have MAD about 70\%, much larger than all other data and these are from Garimella et al. [41]. The figure does not indicate any relation between aspect ratio and deviations.

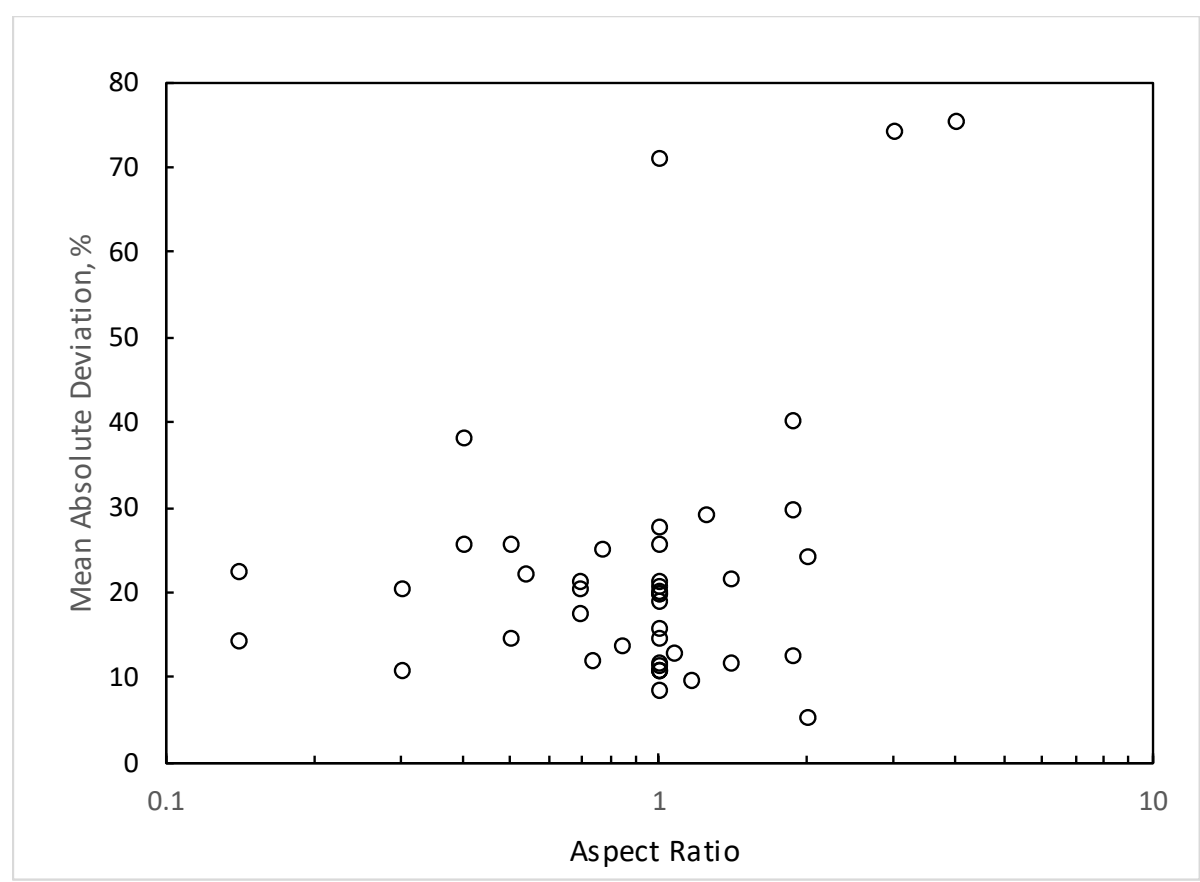

Figure 2. Mean absolute deviations of data sets with Shah [3] correlation vs aspect ratio of channels other than flattened tubes. 
From these observations it may be concluded that the data of Garimella et al. are unique. Their large deviation cannot be attributed to diameter or aspect ratio. The Shah correlation appears to be applicable over the entire range of diameter and aspect ratio.

\subsection{Flattened Tubes}

Table 3 gives the results of comparison of various correlations with the data for flattened tubes. Also included in this table are the results for the round tubes from which the flattened tubes were made. It is seen that some data sets give good agreement with the Shah correlation while some have large deviations. Similar are the results with other correlations. While this may be due to shortcomings of the correlations, there are some inconsistencies in the data which are now pointed out. It is seen that the flattened tube data of Wilson et al. [9] show good agreement with the Shah correlation but their data for round tube has large deviation. In Shah [3] it was shown that this correlation is in good agreement with a very wide range of data for round tubes. Hence lack of agreement with the round tube data of Wilson et al. is unexpected. Kaewon et al. [12] state that their data for round tubes is in good agreement with the correlations of Shah [31] and Akers et al. [36]. During the present analysis it was found that both correlations over-predict the data by around $50 \%$. It seems that there is some error in the figures from which the data was obtained for the present analysis.

The CFD analyses for rectangular and flattened channels show similar behavior. Considering that the Shah correlation gives good agreement with data for channels of rectangular and other shapes, it is rather surprising that its agreement with flattened channel data is not good. It could be that flattened tubes involve some phenomena quite different from those in channels of other shapes or the test data have shortcomings. This matter can be resolved only when more test data become available.

In the following sections, discussions are confined to channels other than flattened tubes except where stated otherwise.

\subsection{Effect of Weber Number}

Weber number is the ratio of inertia force to surface tension force. Surface tension force becomes important at low Weber numbers. According to the Shah [17] correlation, this limit is $\mathrm{We}_{\mathrm{GT}}<100$. Table 4 shows the deviations of various correlations at We $\mathrm{GT}_{\mathrm{G}}$ less than and greater than 100 . It is seen that the MAD of all general correlations except that of Shah are much higher for $\mathrm{We}_{\mathrm{GT}}<100$ than at We $_{\mathrm{GT}}>100$. For example, the MAD of Kim and Mudawar correlation increases from 19.5 to $32.6 \%$ and that of Dorao and Fernandino from 19.5 to $40 \%$. This confirms that $\mathrm{We}_{\mathrm{GT}}>100$ is the limit for the applicability of macro channel correlations for condensation inside channels.

\subsection{Accuracy of Various Correlations}

The Shah correlation gives good agreement over the entire range of data for all shapes. The correlations of Kim and Mudawar, Ananiev et al., and Dorao and Fernandino give fairly good agreement with data for $\mathrm{We}_{\mathrm{GT}}>100$. The correlations of Wen et al. [7], Solanki and Kumar [13] and Kim et al. [10] were based on data for flattened tubes. They perform poorly for all shapes including flattened tubes. The correlation of Solanki and Kumar gives large deviations even with their own data.

Figures 3-7 show the comparison of some test data with various correlations. 


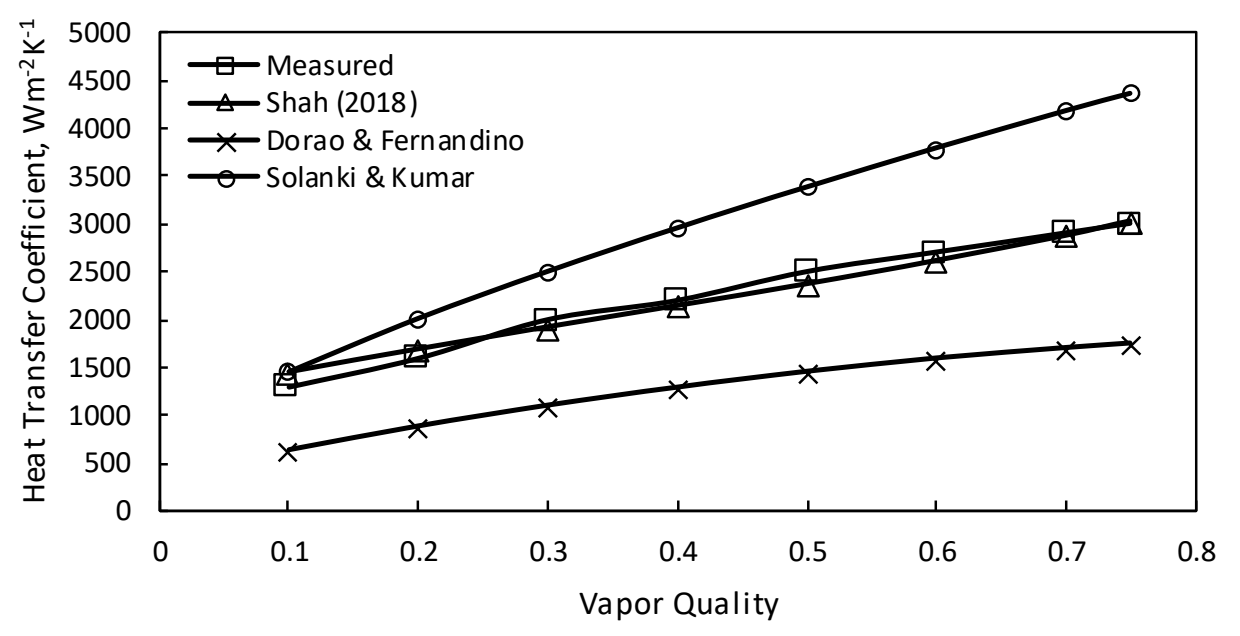

Figure 3. Comparison of the data of Derby et al. [51] in a semi-circular channel with various correlations. $\mathrm{T}_{\mathrm{SAT}}=35 \mathrm{~K}, \mathrm{G}=75 \mathrm{kgm}^{-2} \mathrm{~s}^{-1}, \mathrm{We}_{\mathrm{GT}}=19$.

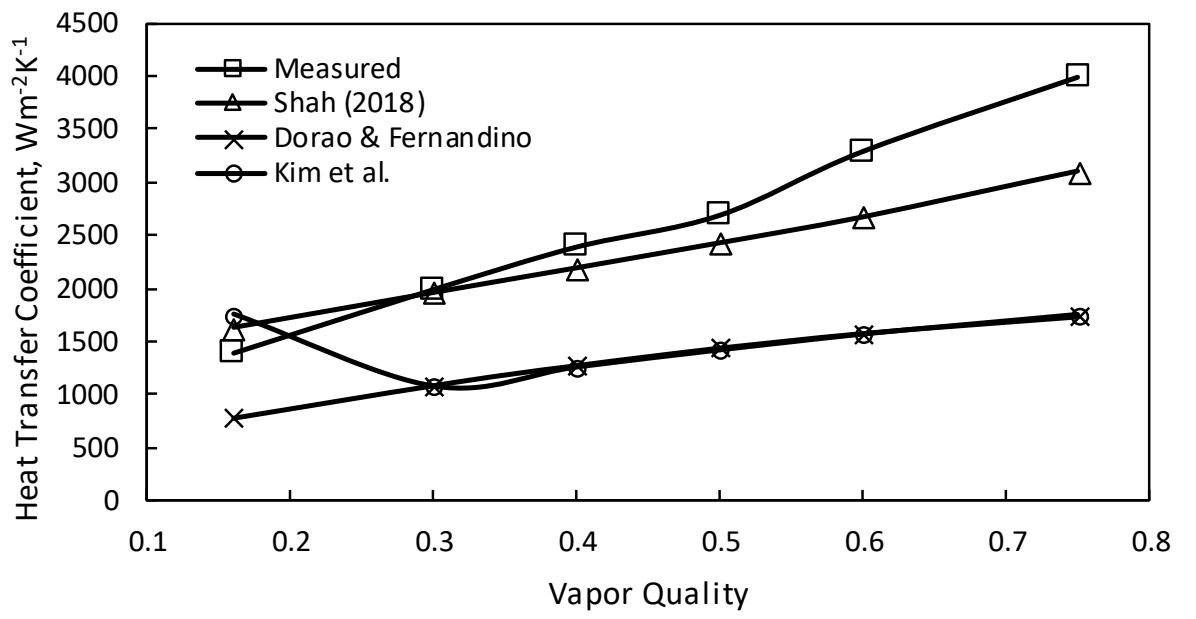

Figure 4. Data of Derby et al. for a triangular channel compared to various correlations. $\mathrm{T}_{\mathrm{SAT}}=35 \mathrm{C}$, $\mathrm{G}=75 \mathrm{kgm}^{-2} \mathrm{~s}^{-1}, \mathrm{We}_{\mathrm{GT}}=19$. 


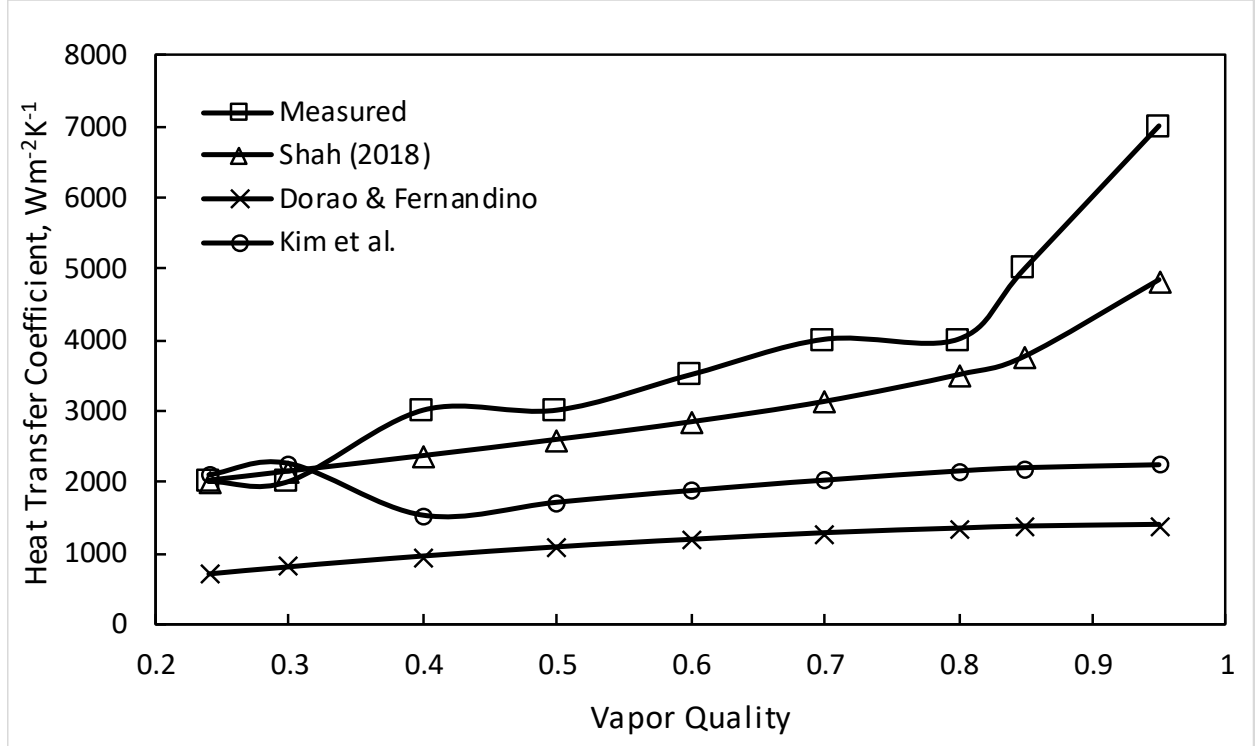

Figure 5. Data of Rahman et al. [30] in a rectangular channel compared to various correlations. $\mathrm{D}_{\mathrm{HYD}}=0.81 \mathrm{~mm}, \mathrm{AR}=0.5, \mathrm{G}=50 \mathrm{kgm}^{-2} \mathrm{~s}^{-1}, \mathrm{~T}_{\mathrm{SAT}}=35 \mathrm{C}$.

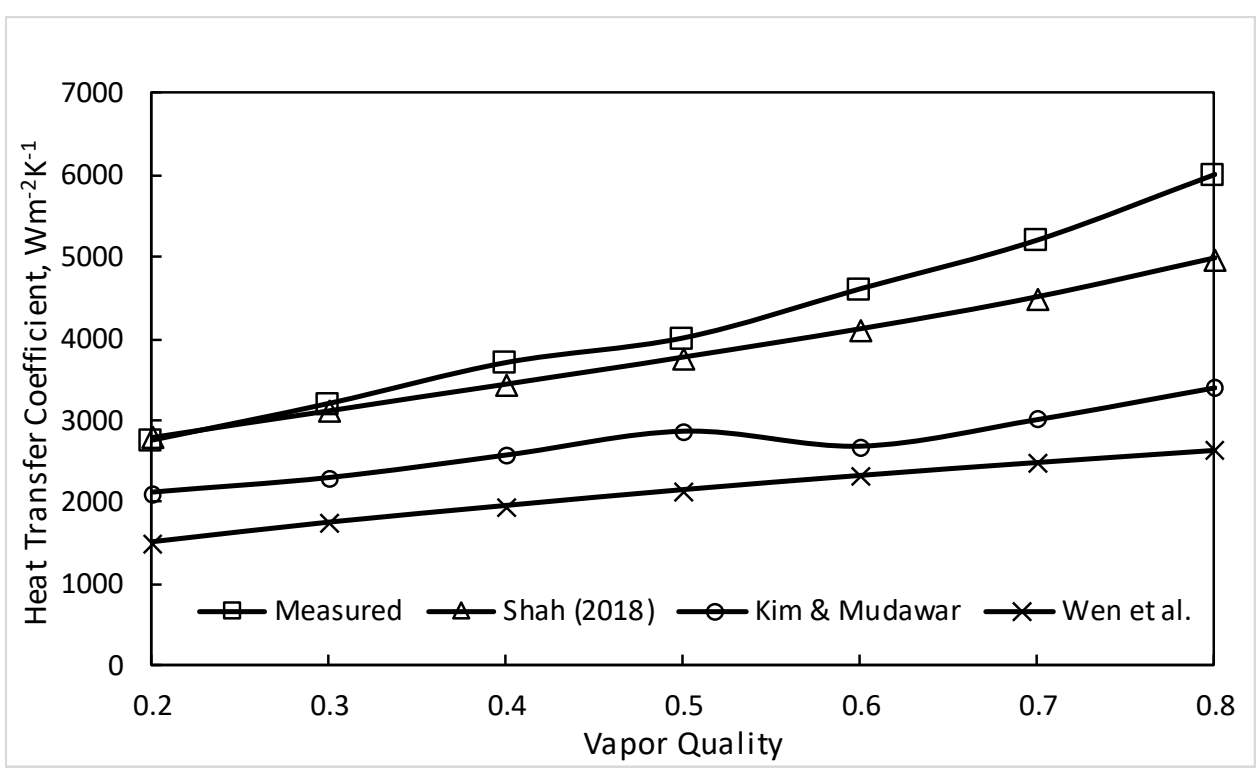

Figure 6. Comparison of the data of Del Col et al. [50] for R-32 in a square channel with various correlations. $D_{\mathrm{HYD}}=1.23 \mathrm{~mm}, \mathrm{G}=100 \mathrm{kgm}^{-2} \mathrm{~s}^{-1}, \mathrm{~T}_{\mathrm{SAT}}=40 \mathrm{C}$. 


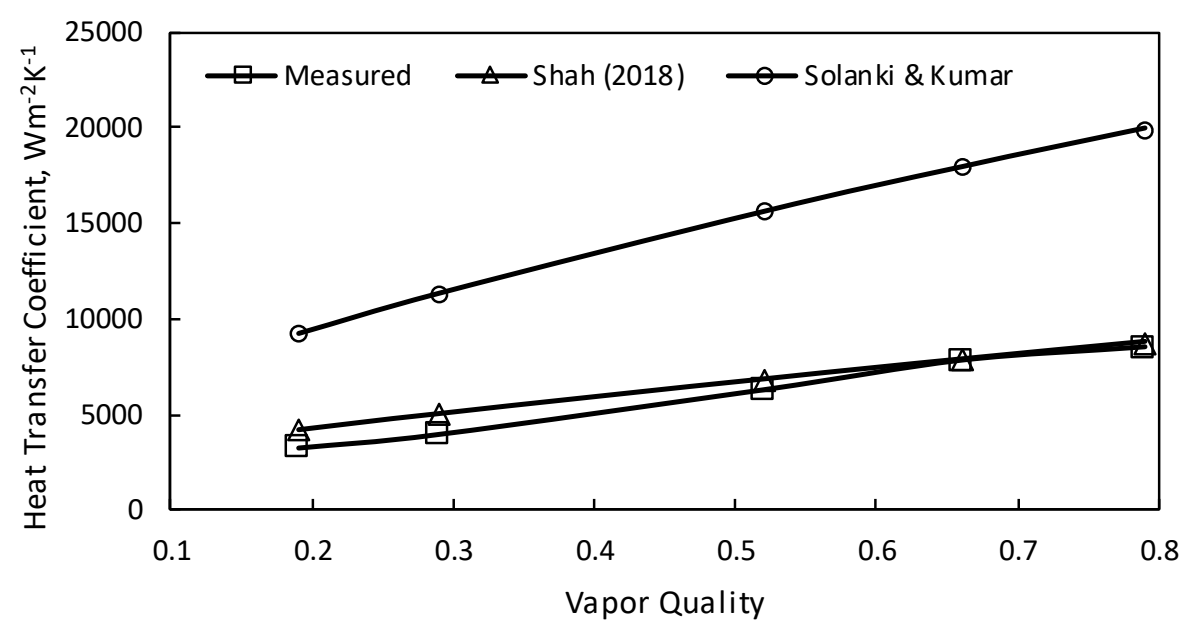

Figure 7. Comparison of the data of Agarwal et al. [46] for barrel shape channels with various correlations. $\mathrm{T}_{\mathrm{SAT}}=55 \mathrm{C}, \mathrm{G}=600 \mathrm{kgm}^{-2} \mathrm{~s}^{-1}$.

\subsection{Effect of Channel Shape}

The Shah correlation shows good agreement with data for square, rectangle, triangle, semi-circle, drum, N, and W shape channels. All these channels have sharp corners. It was shown in Shah [3] that it also gives good agreement with round tube data over a wide range. This correlation does not have any factor for the effect of corners. Hence its agreement with both circular and non-circular channels seems to contradict the results of CFD analyses such as those of Wang and Rose [5-7] which indicate that heat transfer is higher in channels with corners compared to round channels.

\subsection{Effect of Various Parameters}

The present study included 15 fluids. In Shah [3], this correlation was validated with data for 43 fluids in round and non-circular channels. Hence the Shah [3] correlation can be expected to all those fluids for non-circular channels also.

The range of reduced pressure, $\mathrm{We}_{\mathrm{GT}}$ and $\mathrm{Re}_{\mathrm{LT}}$ in the data analyzed in Shah [3] was wider than in the present study. Caution should be exercised in using the Shah [3] correlation beyond their range in the present data analysis when applying to non-circular channels.

\subsection{Complexity of Correlations}

Appendix A gives details of the correlations of Kim and Mudawar, Ananiev et al. and Dorao and Fernadino. The other correlations that were evaluated are already described in the text. It is seen that all these correlations are quite simple except for that of Kim and Mudawar which is quite complex. However, the complexity is not such that it may cause any difficulty in computerized calculations. The Shah correlation is also somewhat complex but simple compared to the Kim and Mudawar correlation.

\subsection{Design Recommendations}

The Shah correlation is recommended for all channel shapes except flattened tubes. No recommendations are made for flattened tubes. 


\section{Conclusions}

1. A number of correlations for condensation in channels were compared to wide-ranging data for non-circular channels.

2. The Shah [3] correlation gave good agreement with data for channels of square, rectangular, triangular, semi-circular, drum, N, and W shapes, MAD being 20.3\% for 1120 data points for 15 fluids from 22 sources. Other general correlations gave fairly good agreement when $\mathrm{We}_{\mathrm{GT}}>100$ but had large deviations when $\mathrm{We}_{\mathrm{GT}}<100$.

3. Data for flattened tubes from five sources were also compared to the same correlations. None of the correlations, including those based on flattened tube data, gave satisfactory agreement. More experimental studies on flattened tubes are needed.

Funding: This research received no external funding.

Acknowledgments: This work did not receive any financial support from any source.

Conflicts of Interest: The author declares no conflict of interest.

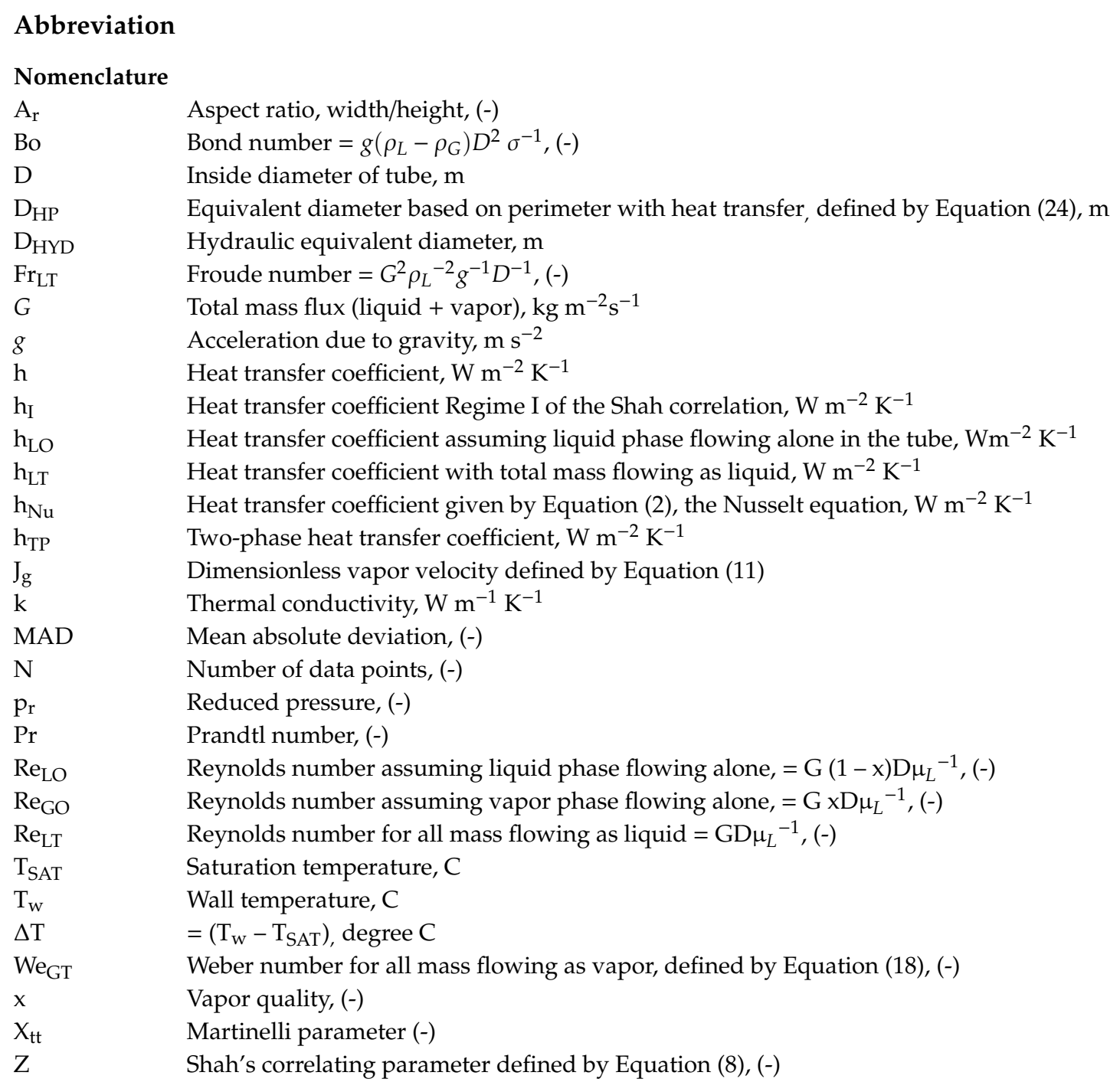




\section{Greek}

$\delta_{\text {avg }}$

$\delta_{\mathrm{m}}$

Average deviation, defined by Equation (24), (-)

$\mu$

Mean absolute deviation, defined by Equation (23), (-)

$\rho \quad$ Density, $\mathrm{kg} \mathrm{m}^{-3}$

$\Sigma \quad$ Mathematical symbol for summation

$\sigma \quad$ Surface tension, $\mathrm{Nm}^{-1}$

\section{Subscripts}

G Vapor

L Liquid

round Round tube

\section{Appendix A}

Ananiev et al. [37] gave the following correlation.

$$
h_{T P}=h_{L T}\left(\frac{\rho_{f}}{\rho_{m}}\right)^{0.5}
$$

where $\rho_{\mathrm{m}}$ is the density of vapor-liquid mixture calculated by the homogeneous model as:

$$
\rho_{m}=\frac{\rho_{f} \rho_{g}}{\rho_{g}+x\left(\rho_{f}-\rho_{g}\right)}
$$

Dorao and Fernandino [2] have given the following correlation.

$$
\begin{gathered}
h_{1}=0.023\left(\operatorname{Re}_{L O}+\operatorname{Re}_{G O}\right)^{0.8} \operatorname{Pr}_{T P}^{0.3} k_{f} / D \\
h_{2}=41.5 D^{0.6}\left(\operatorname{Re}_{L O}+\operatorname{Re}_{G O}\right)^{0.4} \operatorname{Pr}_{T P}^{0.3} k_{f} / D \\
h_{T P}=\left(h_{1}^{9}+h_{2}^{9}\right)^{1 / 9} \\
\operatorname{Pr}_{T P}=\operatorname{Pr}_{f}(1-x)+\operatorname{Pr}_{g} x
\end{gathered}
$$

Equations (A1)-(A4) is dimensional; D used in it should be in meter. For non-circular channels, D is to be replaced by hydraulic equivalent diameter.

Kim and Mudawar [1] developed the following correlation.

For annular flow (smooth annular, wavy-annular, transition) where $\mathrm{We}^{*}>7 \mathrm{X}_{\mathrm{tt}}{ }^{2}$ :

$$
\frac{h_{T P} D}{k_{f}}=0.048 R e_{L O}^{0.69} \operatorname{Pr}_{f}^{0.34} \frac{\phi_{g}}{X_{t t}}
$$

For slug and bubbly flow where $\mathrm{We}^{*}<7 \mathrm{X}_{\mathrm{tt}}{ }^{2}$,

$$
\begin{gathered}
\frac{h_{T P} D}{k_{f}}=\left[\left(0.048 \operatorname{Re}_{L O}^{0.69} \operatorname{Pr}_{f}^{0.34} \frac{\phi_{g}}{X_{t t}}\right)^{2}+\left(3.2 \times 10^{-7} \operatorname{Re}_{L O}^{-0.38} S u_{g}^{1.39}\right)^{2}\right]^{0.5} \\
\phi_{g}^{2}=1+C X++X^{2} \\
X^{2}=\frac{(d p / d z)_{f}}{(d p / d z)_{g}} \\
-\left(\frac{d p}{d z}\right)_{f}=\frac{2 f_{f} G^{2}(1-x)^{2}}{\rho_{f} D} \\
-\left(\frac{d p}{d z}\right)_{g}=\frac{2 f_{g} G^{2} x^{2}}{\rho_{f g} D}
\end{gathered}
$$


For $\operatorname{Re}_{\mathrm{k}}<2000$

$$
f_{k}=16 R e_{k}^{-1}
$$

For $\operatorname{Re}_{\mathrm{k}}=2000$ to 20,000 ,

$$
f_{k}=0.079 R e_{k}^{-0.25}
$$

For $\operatorname{Re}_{\mathrm{k}}>20,000$,

$$
f_{k}=0.046 R e_{k}^{-0.2}
$$

For laminar flow in rectangular channel.

$$
f_{k} R e_{k}=24\left(1-1.3553 \beta+1.9467 \beta^{2}-1.7012 \beta^{3}+0.9564 \beta^{4}-0.2537 \beta^{5}\right)
$$

$\beta$ is the aspect ratio of channel; use its reciprocal if $\beta>1$.

The subscript $\mathrm{k}$ denotes for liquid or vapor.

$$
\begin{aligned}
& \text { For } \operatorname{Re}_{\mathrm{LO}} \geq 2000, \operatorname{Re}_{\mathrm{GO}} \geq 2000, C=0.39 R e_{L T}^{0.03} S u_{g}^{0.1}\left(\frac{\rho_{f}}{\rho_{g}}\right)^{0.35} \\
& \text { For } \operatorname{Re}_{\mathrm{LO}} \geq 2000, \operatorname{Re}_{\mathrm{GO}}<2000, C=0.00087 R e_{L T}^{0.17} S u_{g}^{0.5}\left(\frac{\rho_{f}}{\rho_{g}}\right)^{0.14} \\
& \text { For } \operatorname{Re}_{\mathrm{LO}}<2000, \operatorname{Re}_{\mathrm{GO}} \geq 2000, C=0.0015 R e_{L T}^{0.59} S u_{g}^{0.19}\left(\frac{\rho_{f}}{\rho_{g}}\right)^{0.36} \\
& \text { For } \operatorname{Re}_{\mathrm{LO}}<2000, \operatorname{Re}_{\mathrm{GO}}<2000, C=0.000035 R e_{L T}^{0.44} S u_{g}^{0.5}\left(\frac{\rho_{f}}{\rho_{g}}\right)^{0.48}
\end{aligned}
$$

In the above equations, $\mathrm{D}_{\mathrm{HYD}}$ is used in place of $\mathrm{D}$ for non-circular channels. The modified Weber number $\mathrm{We}^{*}$ is given by:

$$
\begin{gathered}
\text { For } \operatorname{Re}_{\mathrm{LO}}<1250, W e^{*}=2.45 \frac{R e_{G O}^{0.64}}{S u_{g}^{0.3}\left(1+1.09 X_{t t}^{0.039}\right)^{0.4}} \\
\text { For } \operatorname{Re}_{\mathrm{LO}}>1250, W e^{*}=0.85 \frac{R e_{G O}^{0.79} X_{t t}^{0.157}}{S u_{g}^{0.3}\left(1+1.09 X_{t t}^{0.039}\right)^{0.4}}\left[\left(\frac{\mu_{g}}{\mu_{f}}\right)^{2}\left(\frac{\rho_{f}}{\rho_{g}}\right)\right]^{0.084}
\end{gathered}
$$

$\mathrm{Su}_{\mathrm{g}}$ is the Sugomel number defined as,

$$
S u_{g}=\frac{\rho_{g} \sigma D}{\mu_{g}^{2}}
$$

For channels heated only on three sides, heat transfer coefficient calculated by the foregoing is corrected as follows.

$$
h_{T P}=\left(\frac{N u_{3}}{N u_{4}}\right) h_{T P, \text { circular }}
$$

where $\mathrm{h}_{\mathrm{TP}, \text { circular }}$ is the heat transfer coefficient for a circular tube calculated by the foregoing equations.

$$
\begin{gathered}
N u_{3}=\left(8.235\left(1-1.833 \beta+3.767 \beta^{2}-5.814 \beta^{3}+5.361 \beta^{4}-2.0 \beta^{5}\right)\right) \\
N u_{4}=\left(8.235\left(1-2.042 \beta+33.085 \beta^{2}-2.477 \beta^{3}+1.058 \beta^{4}-0.186 \beta^{5}\right)\right)
\end{gathered}
$$

\section{References}

1. Kim, S.; Mudawar, I. Universal approach to predicting heat transfer coefficient for condensing mini/micro-channel flow. Int. J. Heat Mass Transf. 2013, 56, 238-250. [CrossRef]

2. Dorao, C.A.; Fernandino, M. Simple and general correlation for heat transfer during flow condensation inside plain pipes. Int. J. Heat Mass Transf. 2008, 122, 290-305. [CrossRef]

3. Shah, M.M. Improved correlation for heat transfer during condensation in conventional and mini/micro channels. Int. J. Refrig. 2018, 98, 222-237. [CrossRef] 
4. Wang, H.S.; Rose, J.W. A theory of film condensation in horizontal noncircular section microchannels. ASME J. Heat Transf. 2005, 127, 1096-1105. [CrossRef]

5. Wang, H.S.; Rose, J.W. Film condensation in horizontal microchannels: Effect of channel shape. Int. J. Therm. Sci. 2006, 45, 1205-1212. [CrossRef]

6. Wang, H.S.; Rose, J.W. Theory of heat transfer during condensation in microchannels. Int. J. Heat Mass Transf. 2011, 54, 2525-2534. [CrossRef]

7. Wen, J.; Gu, X.; Wang, S.; Li, Y.; Tu, J. Numerical investigation on condensation heat transfer and pressure drop characteristics of R134a in horizontal flattened tubes. Int. J. Refrig. 2018, 85, 441-461. [CrossRef]

8. Zhang, J.; Li, W.; Sherif, S.A. A numerical study of condensation heat transfer and pressure drop in horizontal round and flattened minichannels. Int. J. Therm. Sci. 2016, 106, 80-93. [CrossRef]

9. Wilson, M.J.; Newell, T.A.; Chato, J.C.; Infante Ferreira, C.A. Refrigerant charge, pressure drop, and condensation heat transfer in flattened tubes. Int. J. Refrig. 2003, 16, 442-451. [CrossRef]

10. Kim, N.H.; Lee, E.J.; Byun, H.W. Condensation heat transfer and pressure drop in flattened smooth tubes having different aspect ratio. Exp. Therm. Fluid Sci. 2013, 46, 245-253. [CrossRef]

11. Darzi, M.; Akhavan-Behabadi, M.A.; Sadoughi, M.K.; Razi, P. Experimental study of horizontal flattened tubes performance on condensation of R600a vapor. Int. Commun. Heat Mass Transf. 2015, 62, 18-25. [CrossRef]

12. Kaewon, J.; Naphattharanum, N.; Binmud, R.; Wongwises, S. Condensation heat transfer characteristic of R134a flowing inside mini circular and flattened tubes. Int. J. Heat Mass Transf. 2016, 102, 86-97. [CrossRef]

13. Solanki, A.K.; Kumar, R. Condensation heat transfer and pressure drop characteristics of R-134a inside the flattened tubes at high mass flux and different saturation temperature. Exp. Heat Transf. 2018, 129, 535-548. [CrossRef]

14. Del Col, D.; Bortolin, S.; Cavallini, A.; Matkovic, M. Effect of cross sectional shape during condensation in a single square minichannel. Int. J. Heat Mass Transf. 2011, 54, 3909-3920. [CrossRef]

15. Matkovic, M.; Cavallini, A.; Del Col, D.; Rossetto, L. Experimental study on condensation heat transfer inside a single circular minichannel. Int. J. Heat Mass Transf. 2009, 52, 2311-2323. [CrossRef]

16. Kandlikar, S.G. Fundamental issues related to flow boiling in minichannels and microchannels. Exp. Therm. Fluid Sci. 2002, 26, 389-407. [CrossRef]

17. Shah, M.M. Applicability of correlations for boiling/condensing in macrochannels to minichannels. Heat Mass Transf. Res. J. 2018, 2, 22-32.

18. Da Riva, E.; Del Col, D.; Garimella, S.V.; Cavallini, A. The importance of turbulence during condensation in a horizontal circular minichannel. Int. J. Heat Mass Transf. 2012, 55, 3470-3481. [CrossRef]

19. Rohsenow, W.M.; Webber, J.H.; Ling, A.T. Effect of vapor velocity on laminar and turbulent film condensation. Transf. ASME 1956, 78, 1637-1643.

20. Kim, S.M.; Mudawar, I. Theoretical model for annular flow condensation in rectangular micro-channels. Int. J. Heat Mass Transf. 2012, 55, 958-970. [CrossRef]

21. Kharangate, C.R.; Mudawar, I. Review of computational studies on boiling and condensation. Int. J. Heat Mass Transf. 2017, 108, 1164-1196. [CrossRef]

22. Dobson, M.K.; Chato, J.C. Condensation in smooth horizontal tubes. J. Heat Transf. 1998, 120, $193-213$. [CrossRef]

23. Thome, J.R.; Hajal, J.E.; Cavallini, A. Condensation in horizontal tubes, part 2: New heat transfer model based on flow regimes. Int. J. Heat Mass Transf. 2003, 46, 3365-3387. [CrossRef]

24. Cavallini, A.; Del Col, D.; Doretti, L.; Matkovic, M.; Rossetto, L.; Zilio, C. Condensation in horizontal smooth tubes: A new heat transfer model for heat exchanger design. Heat Transf. Eng. 2006, 27, 31-38. [CrossRef]

25. Shah, M.M. An improved and extended general correlation for heat transfer during condensation in plain tubes. HVAC R Res. 2009, 15, 889-913. [CrossRef]

26. Shah, M.M. General correlation for heat transfer during condensation in plain tubes: Further development and verification. ASHRAE Transf. 2013, 119, 3-11.

27. Awad, M.M.; Dalkilic, A.S.; Wongwises, S. A critical review on condensation heat transfer in microchannels and minichannels. J. Nanotechnol. Sci. Med. 2014, 5, 010904. [CrossRef] 
28. Del Col, D.; Bortolin, S.; Das Riva, E. Predicting methods and numerical modeling of condensation in microchannels. In Encyclopedia of Two-Phase Heat Transfer and Fluid Flow II-Special Topics and Applications; Volume 3: Special Topics in Condensation; World Scientific Publication Co.: Hackensack, NJ, USA, 2015; pp. 37-84.

29. Jige, D.; Inoue, N.; Koyama, S. Condensation of refrigerants in a multiport tube with rectangular minichannels. Int. J. Refrig. 2016, 67, 202-213. [CrossRef]

30. Rahman, M.M.; Kariya, K.; Miyara, A. An experimental study and development of new correlation for condensation heat transfer coefficient of refrigerant inside a multiport minichannel with and without fins. Int. J. Heat Mass Transf. 2018, 116, 50-60. [CrossRef]

31. Keinath, B.L.; Garimella, S. High-pressure condensing refrigerant flows through microchannels, part ii: Heat transfer models. Heat Transf. Eng. 2018. [CrossRef]

32. Shah, M.M. A general correlation for heat transfer during film condensation inside pipes. Int. J. Heat Mass Transf. 1979, 22, 547-556. [CrossRef]

33. Shah, M.M. A new correlation for heat transfer during condensation in horizontal mini/micro channels. Int. J. Refrig. 2016, 64, 187-202. [CrossRef]

34. Shah, M.M. Comprehensive correlations for heat transfer during condensation in conventional and mini/micro channels in all orientations. Int. J. Refrig. 2016, 67, 22-41. [CrossRef]

35. McAdams, W.H. Heat Transmission, 3rd ed.; McGraw Hill: New York, NY, USA, 1954.

36. Akers, W.W.; Deans, H.A.; Crosser, O.K. Condensing heat transfer within horizontal tubes. Chem. Eng. Prog. Symp. Ser. 1959, 59, 171-176.

37. Ananiev, E.P.; Boyko, I.D.; Kruzhilin, G.I. Heat transfer in the presence of steam condensation in horizontal tubes. Int. Dev. Heat Transf. 1961, 2, 290-295.

38. Moser, K.W.; Webb, R.L.; Na, B. A new equivalent Reynolds number model for condensation in smooth tubes. J. Heat Transf. 1998, 120, 410-416. [CrossRef]

39. Dong, T.; Yang, Z. Measurement and modeling of R141b condensation heat transfer in silicon rectangular microchannels. J. Micromech. Microeng. 2008, 18, 085012. [CrossRef]

40. Fronk, B.M.; Garimella, S. Condensation of ammonia and high-temperature-glide ammonia/water zeotropic mixtures in minichannels_Part I: Measurements. Int. J. Heat Mass Transf. 2016, 101, 1343-1356. [CrossRef]

41. Garimella, S.; Agarwal, A.; Fronk, B.M. Condensation heat transfer in rectangular microscale geometries. Int. J. Heat Mass Transf. 2016, 100, 98-110. [CrossRef]

42. Nakashita, K. Study on Condensation of Pure Refrigerant R134a in Multi-Port Extruded Tubes. Ph.D. Thesis, Kyushu University, Fukuoka, Japan, 2002. (In Japanese).

43. Al-Zaidi, A.H.; Mahmoud, M.M.; Karayiannis, T.G. Condensation flow patterns and heat transfer in horizontal microchannels. Exp. Therm. Fluid Sci. 2018, 90, 153-173. [CrossRef]

44. Liu, N.; Xiao, H.; Li, J. Experimental investigation of condensation heat transfer and pressure drop of propane, R1234ze(E) and R22 in minichannels. Appl. Therm. Eng. 2016, 102, 63-72. [CrossRef]

45. Park, J.E.; Vakili-Farhani, F.; Thome, J.R. Experimental study on condensation heat transfer in vertical minichannels for new refrigerant R1234ze(E) versus R134a and R236fa. Exp. Therm. Fluid Sci. 2011, 35, 442-454. [CrossRef]

46. Agarwal, A.; Bandhauer, T.M.; Garimella, S. Measurement and modeling of condensation heat transfer in non-circular microchannels. Int. J. Refrig. 2010, 33, 1169-1179. [CrossRef]

47. Belchí, A.L.; Gómez, F.I.; Cascales, J.R.G.; García, F.V. Condensing two-phase pressure drop and heat transfer coefficient of propane in a horizontal multiport mini-channel tube: Experimental measurements. Int. J. Refrig. 2016, 68, 59-75. [CrossRef]

48. Shin, J.S.; Kim, M.H. An experimental study of flow condensation heat transfer inside circular and rectangular mini-channels. In Proceedings of the Second International Conference on Microchannels and Minichannels, Rochester, NY, USA, 17-19 June 2004; pp. 633-640.

49. Liu, N.; Li, J.M.; Wang, H.S. Heat transfer and pressure drop during condensation of R152a in circular and square microchannels. Exp. Therm. Fluid Sci. 2013, 47, 60-66. [CrossRef]

50. Del Col, D.; Bortalato, M.; Bortolin, S. Comprehensive experimental investigation of two-phase heat transfer and pressure drop with propane in a minichannel. Int. J. Refrig. 2014, 47, 66-84. [CrossRef]

51. Derby, M.; Lee, H.J.; Peles, Y.; Jensen, M.K. Condensation heat transfer in square, triangular, and semi-circular mini-channels. Int. J. Heat Mass Transf. 2012, 55, 187-197. [CrossRef] 
52. Cavallini, A.; Col, D.D.; Doretti, L.; Matkovic, M.; Rosetto, L.; Zilio, C. Condensation heat transfer and pressure gradient inside multiport minichannels. Heat Transf. Eng. 2005, 26, 45-55. [CrossRef]

53. Koyama, S.; Kuwahara, K.; Nakashita, K. Condensation of refrigerant in a multi-port channel. In Proceedings of the First International Conference on Microchannels and Minichannels, Rochester, NY, USA, 24-25 April 2003; pp. 193-205.

54. Al-Hajri, E.; Shooshtari, A.H.; Dessiatoun, S.; Ohadi, M.M. Performance characterization of R134a and R245fa in a high aspect ratio microchannel condenser. Int. J. Refrig. 2013, 36, 588-600. [CrossRef]

55. Wang, W.W.; Radcliff, T.D.; Christensen, R.N. A condensation heat transfer correlation for millimeter-scale tubing with flow regime transition. Exp. Therm. Fluid Sci. 2002, 26, 473-485. [CrossRef]

C 2019 by the author. Licensee MDPI, Basel, Switzerland. This article is an open access article distributed under the terms and conditions of the Creative Commons Attribution (CC BY) license (http://creativecommons.org/licenses/by/4.0/). 